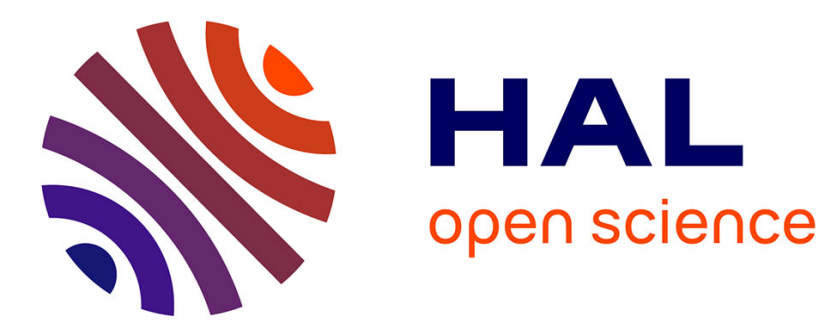

\title{
Deterministic Sensitivity Analysis for a Model for Flow in Porous Media
}

Estelle Marchand, François Clément, Jean E. Roberts, Guillaume Pépin

\section{To cite this version:}

Estelle Marchand, François Clément, Jean E. Roberts, Guillaume Pépin. Deterministic Sensitivity Analysis for a Model for Flow in Porous Media. [Research Report] RR-6502, INRIA. 2008. inria00271986v2

\section{HAL Id: inria-00271986 https://hal.inria.fr/inria-00271986v2}

Submitted on 14 Apr 2008

HAL is a multi-disciplinary open access archive for the deposit and dissemination of scientific research documents, whether they are published or not. The documents may come from teaching and research institutions in France or abroad, or from public or private research centers.
L'archive ouverte pluridisciplinaire HAL, est destinée au dépôt et à la diffusion de documents scientifiques de niveau recherche, publiés ou non, émanant des établissements d'enseignement et de recherche français ou étrangers, des laboratoires publics ou privés. 
INSTITUT NATIONAL DE RECHERCHE EN INFORMATIQUE ET EN AUTOMATIQUE

\section{Deterministic Sensitivity Analysis for a Model for Flow in Porous Media}

Estelle Marchand — François Clément — Jean E. Roberts — Guillaume Pépin

$\mathbf{N}^{\circ} 6502$

April 2008

Thème NUM

\section{apport}

derecherche 



\title{
Deterministic Sensitivity Analysis for a Model for Flow in Porous Media
}

\author{
Estelle Marchand ${ }^{*}{ }^{\dagger}$, François Clément ${ }^{*}$, Jean E. Roberts * , Guillaume Pépin ${ }^{\dagger}$ \\ Thème NUM - Systèmes numériques \\ Équipes-Projets Estime \\ Rapport de recherche $n^{\circ} 6502$ - April $2008-28$ pages
}

\begin{abstract}
A deterministic method for sensitivity analysis is developed and applied to a mathematical model for the simulation of flow in porous media. The method is based on the singular value decomposition (SVD) of the Jacobian matrix of the model. It is a local approach to sensitivity analysis providing a hierarchical classification of the directions in both the input space and of those in the output space reflecting the degree of sensitiveness of the latter to the former. Its low computational cost, in comparison with that of statistical approaches, allows the study of the variability of the results of the sensitivity analysis due to the variations of the input parameters of the model, and thus it can provide a quality criterion for the validity of more classical probabilistic global approaches. For the example treated here, however, this variability is weak, and deterministic and statistical methods yield similar sensitivity results.
\end{abstract}

Key-words: Deterministic Sensitivity Analysis, Singular Value Decomposition, Darcy Flow, Mixed Hybrid Finite Elements, Automatic Differentiation, Nuclear Waste Storage

* Projet Estime.

$\dagger$ ANDRA, DSCS, 92298 Chatenay-Malabry cedex, France.

Centre de recherche INRIA Paris - Rocquencourt

Domaine de Voluceau, Rocquencourt, BP 105, 78153 Le Chesnay Cedex

Téléphone : +33139635511-Télécopie : +33139635330 


\section{Analyse de sensibilité déterministe pour un modèle d'écoulement en milieux poreux}

Résumé : Une méthode déterministe pour l'analyse de sensibilité est développée et appliquée à un modèle mathématique pour la simulation d'écoulements en milieux poreux. La méthode est basée sur la décomposition en valeurs singulières (SVD) de la matrice Jacobienne du modèle. C'est une approche locale d'analyse de sensibilité qui fournit une classification hiérarchique des directions dans les espaces d'entrée et de celles dans l'espace de sortie traduisant le degré de sensibilité de ces dernières sur ces premières. Son coup de calcul faible par rapport à celui des approches statistiques rend possible une étude de la variabilité des résultats en fonction des paramètres d'entrée du modèle, et permet ainsi de fournir un critère de qualité sur la validité des approches probabilistes, plus classiques. Cependant, pour l'exemple considéré ici, cette variabilité est faible et les méthodes déterministe et statistique fournissent des résultats similaires.

Mots-clés : Analyse de sensibilité déterministe, Décomposition en valeurs singulières, Écoulement Darcéen, Éléments finis mixtes hybrides, Différentiation automatique, Stockage de déchets nucléaires 


\section{Introduction}

The questions of safety and uncertainty are central to feasibility studies for the underground storage of nuclear waste. One of the important points to be considered is the problem of the evaluation of uncertainties concerning safety indicators (the "output parameters") due to uncertainties concerning properties of the subsoil around a proposed storage site, properties such as hydraulic conductivity, or properties of the contaminants (the "input parameters"). Uncertainties concerning the input parameters are due to the imprecision of measurement techniques or to spatial variability. The measure of the water flow through specified outlet channels is a natural safety indicator since contaminants are mainly transported by water.

Two different domains are concerned with the quantification of the influence of the input parameters on the safety indicators. One is uncertainty analysis which is concerned with the quantification, in terms of say distributions or quantiles, of the uncertainty concerning the safety indicators. The other is sensitivity analysis which determines weights indicating the degree of influence of particular input parameters on particular safety indicators.

Both uncertainty analysis and sensitivity analysis can be addressed using probabilistic approaches such as Monte-Carlo methods. These methods give good results and are relatively easy to implement, thus their popularity, but they are expensive to use because they require a large number of simulations (see for example [5]). Probabilistic approaches are global in the sense that they take into account all the variations of the input parameters in a given range, but they become less reliable for highly nonlinear problems.

The deterministic method investigated here is much less demanding in terms of computing time, but it gives only local information in the sense that for nonlinear problems the results obtained are relevant only for small variations of the input parameters around some chosen set of input parameters, the size of the permissible variation depending on the degree of the nonlinearity. With this method "first order" uncertainties are computed using the derivatives of the function $F$ that associates the output parameters to the input parameters. The hierarchization of the inputs of the function $F$ according to their influence on the outputs of $F$ is provided by the Singular Value Decomposition (SVD) of the Jacobian matrix $F^{\prime}(x)$ [21, 6, 7, 1]; see also 35] for the history of the SVD and [16] for a detailed description. It is interesting to note that the SVD, the main tool for the deterministic approach described here, is also commonly used in statistical data analysis, see [8, 9, 25, 36].

The probabilistic and deterministic approaches to sensitivity analysis might rightly be seen as complementary, and it is worthwhile to develop both. To our knowledge, the deterministic approach has not yet been used for flow problems in porous media. The main objective here is to present the deterministic method for sensitivity analysis and to show its feasibility for the study of 3D Darcy flow in a realistic test case, and then to compare the results obtained using the deterministic method with those obtained using a Monte Carlo method for the same problem.

When dealing with first order approximations, differentiation is a key issue and different methods can be used for computing $F^{\prime}(x)$ : divided differences, automatic differentiation, analytic differentiation for possibly implicit problems. An explanation of automatic differentiation can be found in 11], an example using automatic differentiation for a huge Fortran code is given in [3] and an example for a C++ code is given in 2. The theory concerning analytic differentiation is developed in [20] and an example may be found in [29. For each method of differentiation, direct mode or reverse mode can be used. (Reverse mode differentiation is equivalent to the differentiation using the adjoint state method.) These derivatives can be used in combination with interval arithmetic to evaluate uncertainties in the form of intervals containing the image under $F$ of input intervals 32 .

In the second section of this paper, we describe probabilistic methods and deterministic methods for sensitivity analysis. In the third section, we give some details about the computation of the derivatives used for the deterministic analysis. In the fourth section, we present a numerical model for flow in porous media. In the fifth section, we compare numerical results obtained using a probabilistic analysis with those obtained using a deterministic analysis for a realistic example representing water flow around a potential nuclear waste storage site. 


\section{Sensitivity analysis}

We consider a general model $F: x \in \mathbb{R}^{n_{\mathrm{ip}}} \mapsto y \in \mathbb{R}^{n_{\mathrm{op}}}$ with $n_{\mathrm{ip}}, n_{\mathrm{op}} \in \mathbb{N}^{\star}$.

\subsection{Probabilistic analysis}

In this work, the global probabilistic analysis is carried out using a Monte-Carlo method. This method consists in examining the influence of the uncertainty about input data on the uncertainty about the output, where the uncertainties have been quantified say in terms of variance. This study covers the global variation of the values through sampling.

First, the uncertainty about scalar components of the input data is defined with Probabilistic Density Functions (PDF's), taking into account various kinds of uncertainties such as those representing natural variability, those coming from up-scaling, or those due to imprecision in the measurements. The choice of laws for defining the PDF's is a matter for specialists. These laws generally include correlations and constraints between variables. In other words the components of the input data are not necessarily independent. A relation between two parameters may be better known than the individual parameters, e.g. for continuous input parameters, the "heterogeneity" of the parameter may be bounded. In this case, PDF's can be applied to relations between input parameters. In practice, we will consider a basis of variables, which we assume to be mutually independent and which may or may not correspond to input parameters. A PDF is defined for each of the basis variables, and each input parameter is explicitly defined in terms of the basis variables.

Next, various samples of input data are generated. There are several methods available for this. We used Latin Hypercube Sampling (LHS) (see for example 14]). Let $N$ be the desired number of samples. For each of the $n_{\mathrm{ip}}^{\prime} \leq n_{\mathrm{ip}}$ basis variables, the set of possible values is divided into $N$ equiprobable intervals and one value is randomly picked from each of these $N$ intervals. The values selected for the $n_{\mathrm{ip}}^{\prime}$ components are randomly combined, to form $N$ samples of the basis variables (and then of the input parameters) in such a way that for each of the basis variables, each selected value appears in precisely one sample. LHS is a compromise between random sampling ( $N$ samples are randomly selected, accordantly with the PDF), which may fail to represent the extreme possible values, and stratified sampling, in which the $n_{\mathrm{ip}}^{\prime}$ - dimensional set of possible input parameters must be divided into equiprobable subsets (a process which can be difficult) before randomly picking one sample from each subset. The $n_{\text {ip }} \times N$ matrix formed from the samples of input parameters is denoted by $x=\left(x^{1}, \ldots, x^{N}\right)$, with, for $n=1, \ldots, N, x^{n} \in \mathbb{R}^{n_{\mathrm{ip}}}$.

Samples of output parameters are computed by applying $F$ to each generated sample. They form the $n_{\mathrm{op}} \times N$ matrix $y=\left(y^{1}, \ldots, y^{N}\right)$, with, for $n=1, \ldots, N, y^{n} \in \mathbb{R}^{n_{\mathrm{op}}}$ and $y^{n}=F\left(x^{n}\right)$. The result of a sensitivity analysis is a representation of the relations existing between input parameters and output parameters. These relations can be quantified by various statistical indicators. The simpler indicators are by construction meaningful only for smooth functions. Some transformations can be performed to deal with more irregular functions but there is no "universal" indicator (further details about these indicators may be found in [15]):

- One can compute correlation coefficients between individual components of input and output data (Pearson coefficients)

$$
\operatorname{cor}\left(x_{i}, y_{j}\right)=\frac{\sum_{n=1}^{N}\left(x_{i}^{n}-\bar{x}_{i}\right)\left(y_{j}^{n}-\bar{y}_{j}\right)}{\sqrt{\sum_{n=1}^{N}\left(x_{i}^{n}-\bar{x}_{i}\right)^{2}} \sqrt{\sum_{n=1}^{N}\left(y_{j}^{n}-\bar{y}_{j}\right)^{2}}},
$$

where $\bar{x}_{i}$ and $\bar{y}_{j}$ are the statistical means for $i=1, \ldots, n_{\mathrm{ip}}$ and $j=1, \ldots, n_{\mathrm{op}}$. These indicators are meaningful when the relations between input data and output data can be approximated by linear (affine) laws. In this case, Pearson coefficients are proportional to the coefficients 
of the linear relation between (centered) input and output data. When the relations between input and output parameters are strongly nonlinear, it can be interesting to replace, for each component of the input or output data, individual values by their ranks in the sampling of the considered component, the ranks being defined by

$$
\begin{array}{lll}
\text { for } i=1, \ldots, n_{\mathrm{ip}}, & n=1, \ldots, N, & r_{x_{i}}^{n}=\operatorname{card}\left\{m \in 1, \ldots, N, x_{i}^{m} \geq x_{i}^{n}\right\}, \\
\text { for } j=1, \ldots, n_{\mathrm{op}}, & n=1, \ldots, N, & r_{y_{j}}^{n}=\operatorname{card}\left\{m \in 1, \ldots, N, y_{j}^{m} \geq y_{j}^{n}\right\} .
\end{array}
$$

Correlation coefficients can be computed for these transformed data. The Spearman correlation coefficient measures the monotonicity between the uncertainty of the result and the uncertainty of input data. It is the Pearson coefficient computed for the ranks: $\operatorname{spear}\left(x_{i}, y_{j}\right)=$ $\operatorname{cor}\left(r_{x_{i}}, r_{y_{j}}\right)$ for $i=1, \ldots, n_{\mathrm{ip}}, j=1, \ldots, n_{\mathrm{op}}$.

- These coefficients can be corrected to reduce the effect of other input components when quantifying the relation between particular input and output components. Linear regressions must first be computed following

$$
\begin{aligned}
& \hat{x}_{i}=c_{0}+\sum_{\substack{k=1, k \neq i \\
n_{\text {ip }}}}^{n_{\text {ip }}} c_{k} x_{k} \\
& \hat{y}_{j \mid i}=b_{0 \mid i}+\sum_{k=1, k \neq i} b_{k \mid i} x_{k},
\end{aligned}
$$

where the coefficients $c_{k}, b_{k \mid i}, k=1, \ldots, i-1, i+1, \ldots, n_{i p}$, dependent on $i$ and $j$, minimize the quadratic errors over the samples $\sum_{n=1}^{N}\left(x_{i}^{n}-\hat{x}_{i}^{n}\right)^{2}$ and $\sum_{n=1}^{N}\left(y_{j}^{n}-\hat{y}_{j \mid i}^{n}\right)^{2}$. The Partial Correlation Coefficient (PCC) is then defined by $\operatorname{PCC}\left(x_{i}, y_{j}\right)=\operatorname{cor}\left(x_{i}-\hat{x}_{i}, y_{j}-\hat{y}_{j \mid i}\right)$. The Partial Rank Correlation Coefficient (PRCC) is the PCC computed for the ranks: $\operatorname{PRCC}\left(x_{i}, y_{j}\right)=\operatorname{PCC}\left(r_{x_{i}}, r_{y_{j}}\right)$.

- The Standard Rank Regression Coefficient (SRRC) is related to the effect of modifying an input component by a fixed fraction of its standard deviation. The following regression model is considered (it is not a partial regression)

$$
\hat{y}_{j}=b_{0}+\sum_{k=1}^{n_{\mathrm{ip}}} b_{k} x_{k}
$$

where the coefficients $b_{k}, k=1, \ldots, n_{\text {ip }}$ are chosen to minimize the quadratic error over the samples $\sum_{n=1}^{N}\left(y_{j}^{n}-\hat{y}_{j}^{n}\right)^{2}$. The Standard Regression Coefficient is defined as $\operatorname{SRC}\left(x_{i}, y_{j}\right)=$ $\frac{b_{i} \hat{\sigma}\left(x_{i}\right)}{\hat{\sigma}\left(y_{j}\right)}$, where $\hat{\sigma}$ is the estimator for standard deviation defined as $\hat{\sigma}\left(x_{k}\right)=\frac{1}{\sqrt{N-1}} \sqrt{\sum_{n=1}^{N}\left(x_{k}^{n}-\bar{x}_{k}\right)^{2}}$.

The SRRC is the SRC for the ranks: $\operatorname{SRRC}\left(x_{i}, y_{j}\right)=\operatorname{SRC}\left(r_{x_{i}}, r_{y_{j}}\right)$.

We note that these indicators take values in the interval $[-1,1]$. The nearer the absolute value of an indicator is to 1 , the stronger is the dependence \{input parameter/output parameter\}. The sign of the coefficient depends on the monotonicity of the relation and is positive if the considered input and output components increase together. Small values (e.g. those smaller than 0.2) indicate a very weak dependence. These coefficients make it possible to rank input parameters according to their degree of influence. 


\subsection{Deterministic analysis}

Local deterministic uncertainty analysis is based on a first order approximation: we consider a linearized problem

$$
\delta x \in \mathbb{R}^{n_{\mathrm{ip}}} \mapsto \delta y=F^{\prime}(x) \delta x \in \mathbb{R}^{n_{\mathrm{op}}},
$$

which associates approximate variations of the output parameters $\delta y$ to small variations of the input parameters $\delta x$ around a given set of input parameters $x$. For an affine function $F$, an upper bound on the uncertainty about $y_{i}$, for $i=1, \ldots, n_{\mathrm{op}}$, would be $\sum_{j=1}^{n_{\mathrm{ip}}}\left|F^{\prime}(x)_{i j}\right|\left|\Delta x_{j}\right|$, where the nonnegative value $\left|\Delta x_{j}\right|$ quantifies the uncertainty about the input parameter $x_{j}$. Correlations between input parameters are not taken into account in this upper bound.

Deterministic sensitivity analysis is based on the Singular Value Decomposition (SVD) of the Jacobian matrix $F^{\prime}(x)$ for a given input vector $x$. The SVD of the rectangular matrix $F^{\prime}(x)$ is given by

$$
F^{\prime}(x)=U S V^{T}
$$

where $U$ and $V$ are orthogonal matrices and $S$ is a diagonal matrix of the same size as $F^{\prime}$. The columns of $V$ are called the singular vectors of the input space, and the columns of $U$ are called the singular vectors of the output space. The diagonal terms of $S$ are the singular values of $F^{\prime}$, that is to say the square roots of the eigenvalues of the square symmetric matrix ${F^{\prime}}^{T} F^{\prime}$. They are nonnegative numbers sorted in nonincreasing order.

The SVD performs an orthogonal change of basis in both the input and output spaces. If we denote by $u_{k}$ (respectively $v_{k}$ ) the $k^{t h}$ column of $U$ (respectively of $V$ ) and by $s_{k}$ the $k^{t h}$ diagonal term of $S$, we have

$$
\begin{array}{ll}
\text { for } k \leq \min \left(n_{\mathrm{ip}}, n_{\mathrm{op}}\right), & F^{\prime}(x) v_{k}=s_{k} u_{k} ; \\
\text { if } n_{\mathrm{op}}<n_{\mathrm{ip}}, \text { for } n_{\mathrm{op}}<k \leq n_{\mathrm{ip}}, & F^{\prime}(x) v_{k}=\mathbf{0} .
\end{array}
$$

Then the output variation associated with an input variation $\delta x$ will be

$$
\delta y=\sum_{k=1}^{\min \left(n_{\mathrm{ip}}, n_{\mathrm{op}}\right)} s_{k}\left\langle\delta x, v_{k}\right\rangle u_{k}
$$

where $\langle.,$.$\rangle denotes the canonical scalar product. This means that for k \leq \min \left(n_{\mathrm{ip}}, n_{\mathrm{op}}\right)$, the variation of the output parameters in the direction $u_{k}$ depends exclusively on the variation on the input parameters in the direction $v_{k}$, and this dependence is quantified by the singular value $s_{k}$. If $n_{\mathrm{op}}<n_{\mathrm{ip}}$, for $n_{\mathrm{op}}<k \leq n_{\mathrm{ip}}$, i.e. if $v_{k}$ is in the kernel of $F^{\prime}$, a variation of the input parameters in the direction $v_{k}$ has no influence on the output: for $n_{\mathrm{op}}<k \leq n_{\text {ip }}$ and $\alpha \in \mathbb{R}$, $F^{\prime}(x)\left(\delta x+\alpha v_{k}\right)=F^{\prime}(x) \delta x$. On the other hand, if $n_{\text {ip }}<n_{\text {op }}$, for $n_{\text {ip }}<k \leq n_{\text {op }}$, then $\left\langle\delta y, u_{k}\right\rangle=0$ and $u_{k}$ is in the orthogonal complement of the image of $F^{\prime}(x)$, i.e. the output does not vary in the direction of $u_{k}$.

So the rate of decrease of the singular values provides a hierarchical classification of the influence of the directions $v_{k}$ in the input space on the directions $u_{k}$ in the output space.

SVD is useful in various contexts. For example it can be used to determine which input parameters should be more accurately measured in order to obtain greater precision for particular output parameters. If say the input parameters are the uncertain values of a piecewise constant field, a field constant in each of several zones into which the domain has been divided, then the SVD results can indicate in which zones more precise measurements need to be taken, or which zones should be further divided into more zones in which measurements are taken in order to reduce the spatial variability. In another context, when output parameters can be measured and must be controlled, the SVD results sometimes make it possible to easily identify which input parameters must be modified to alter one particular output parameter without changing the other output parameters. Generally, the associated singular vector in the input space is not parallel to 
one of the main axes, and several input parameters may need to be modified together with the corresponding weights. These possibilities will be illustrated in section 5.3.4.

The SVD algorithm is widely used and several implementations are available. Here the routine dgesvd provided in the linear algebra library LAPACK is used; see [19].

\section{$2.3 \quad$ A $1 \mathrm{D}$ example}

We consider a flow in a one-dimensional porous medium $\Omega=[0, L]$ of permeability $\mathcal{K}$ with no source term

$$
\frac{\partial \Phi}{\partial \xi}=0, \quad \Phi=-\mathcal{K} \frac{\partial p}{\partial \xi},
$$

and with Dirichlet boundary conditions $p=p_{0}$ at $\xi=0, p=p_{L}$ at $\xi=L$. The velocity field $\Phi$ is constant,

$$
\Phi=\frac{p_{0}-p_{L}}{\int_{\xi=0}^{L} \frac{1}{\mathcal{K}(\xi)}} .
$$

If the hydraulic conductivity is assumed to be piecewise constant with $n_{z}$ zones of length $L_{i}$, $i=1, \ldots, n_{z}$, we consider $F(x)=F\left(K_{1}, \cdots, K_{n_{z}}\right)=\Phi$ and the sensitivities are

$$
\frac{\partial \Phi}{\partial K_{i}}=\frac{\Phi^{2}}{p_{0}-p_{L}} \frac{L_{i}}{K_{i}^{2}}
$$

With a logarithmic parametrization, i.e. considering $\hat{F}(\hat{x})=\hat{F}\left(\log K_{1}, \ldots, \log K_{n_{z}}\right)=F\left(K_{1}, \ldots, K_{n_{z}}\right)$, the matrix to be decomposed into singular values is $\hat{F}^{\prime}(\hat{x})=\frac{\Phi^{2}}{p_{0}-p_{L}}\left(\frac{L_{1}}{K_{1}}, \ldots, \frac{L_{n_{z}}}{K_{n_{z}}}\right)$. The only singular value is $s_{1}=\left\|\hat{F}^{\prime}(\hat{x})\right\|$ and the first singular vector in the input space, that is the unit vector orthogonal of the kernel of $\hat{F}^{\prime}(\hat{x})$, is proportional to $\hat{F}^{\prime}(\hat{x})$, and its larger components correspond to the zones of lower permeability. The importance of $K_{i}$ increases also with the length of the zone $L_{i}$. Without logarithmic parametrization, the hierarchy can be reordered except when $L_{1}=\ldots=L_{n_{z}}$. In this case only the rate of decrease is accelerated.

We give an approximation of the theoretic Pearson coefficient for the case in which there are only two zones with $\frac{K_{1}}{L_{1}} \gg \frac{K_{2}}{L_{2}}$. We denote $u_{1}=\frac{K_{1}}{L_{1}}$ and $u_{2}=\frac{K_{2}}{L_{2}}$. In this case, the equation (10) can be approximated by

$$
\operatorname{cor}\left(K_{1}, \Phi\right)=\operatorname{cor}\left(u_{1}, \frac{u_{1} u_{2}}{u_{1}+u_{2}}\right) \approx \operatorname{cor}\left(u_{1}, u_{2}\right)=\operatorname{cor}\left(K_{1}, K_{2}\right)
$$

and

$$
\operatorname{cor}\left(K_{2}, \Phi\right)=\operatorname{cor}\left(u_{2}, \frac{u_{1} u_{2}}{u_{1}+u_{2}}\right) \approx 1
$$

and we conclude again that the lower permeability has the greater impact.

Note that one needs stronger hypotheses to obtain general results with the statistical approach. 


\section{Differentiation}

The first step in deterministic sensitivity analysis is the computation of the Jacobian matrix of the function $F$.

\subsection{Notation}

We suppose that the model $F$ is not a closed-form formula.

Let $\mathcal{P}$ be an open subset of a normed vector space, $n_{\text {dof }}$ a large integer and let

$$
\tilde{F}: \tilde{x} \in \mathcal{P} \mapsto \tilde{y} \in \mathbb{R}^{n_{\text {dof }}}
$$

be an implicit function defined by

$$
\tilde{y}=\tilde{F}(\tilde{x}) \Leftrightarrow E(\tilde{x}, \tilde{y})=\mathbf{0} .
$$

The equation $E(\tilde{x}, \tilde{y})=\mathbf{0}$, the state equation, is say a discretization of a partial derivative equation, with $\tilde{x}$ a discretization of a field for a physical parameter and $\tilde{y}$ the vector of degrees of freedom.

Let $n_{\text {ip }}$ and $n_{\mathrm{op}}$ be integers, at least one of which is small relative to $n_{\text {dof }}$ and let

$$
\begin{aligned}
& P: \quad x \in \mathbb{R}^{n_{\mathrm{ip}}} \mapsto \tilde{x} \in \mathcal{P} \\
& O: \quad \tilde{y} \in \mathbb{R}^{n_{\mathrm{dof}}} \mapsto y \in \mathbb{R}^{n_{\mathrm{op}}}
\end{aligned}
$$

be closed-form formulas. The parametrization operator $P$ serves to reduce the dimension of the input space in order to avoid over-parametrization problems while the observation, or measure, operator $O$ serves to reduce the dimension of the output space in order to sum up the output into indicators or to model experimental measures performed in the real fields. A modular implementation of these operators makes it possible to change them easily for different numerical experiments.

The model to be differentiated is then

$$
F=O \circ \tilde{F} \circ P .
$$

\subsection{Differentiation techniques}

For the problem treated in this article, we investigated several different techniques of differentiation [23] 11, 26]. Approximate differentiation through divided differences is an obvious possibility but this technique is either insufficiently accurate or too expensive in computation time since it is difficult to choose the optimal differentiation step. Hence it is best reserved for validation purposes. Possible exact methods are automatic differentiation and manual differentiation, i.e. differentiation based on analytical formulas.

It is important to be able to differentiate the model either in direct mode (i.e. computation of the Jacobian matrix by columns, which is advantageous when $n_{\mathrm{ip}} \leq n_{\mathrm{op}}$ ) or in reverse mode (i.e. computation of the Jacobian matrix by rows, which is advantageous when $n_{\mathrm{ip}} \geq n_{\mathrm{op}}$ ).

We have to differentiate a function computed using a C++ code both in direct mode and in reverse mode. For this reason we have investigated the tool of automatic differentiation by operator overloading ADOL-C [12. By now, according to [17, other alternatives are available as FADBAD/TADIFF, OpenAD, and YAO.

We compared the performances of ADOL-C with those of manual differentiation. ADOL-C was very competitive with respect to development time, and execution times were comparable to those obtained with manually differentiated codes, but memory management was difficult in reverse mode with ADOL-C for realistic computations. For this reason we used a manually differentiated code and ADOL-C was used for validation (ADOL-C was also competitive with divided differences concerning development times). However solutions to the problems of memory management are proposed in [13; the algorithm described in [10] is available at [18. 


\subsection{Verification of derivative computations}

The most error prone step of the deterministic method for sensitivity analysis is the computation of the Jacobian matrix of $F$. We give in this section some elements concerning the verification of the code computing the derivatives, assuming that the code for the function $F$ itself has already been validated. Details for the example presented in this paper are given in [26].

First of all, at least two different methods must be implemented (among automatic differentiation, manual differentiation, finite differences, each method can be implemented in reverse mode and/or in direct mode).

In order to locate the errors, it is important to be able to verify subfunctions one by one.

The code to be verified computes the derivative of a solver for a partial derivative equation. The input of the solver is the vector of the coefficients of a parameter field occurring in the equation on some discrete basis, and the output is for example a subset of the degrees of freedom of the solver. The operators $O$ and $P$ should be implemented independently of the rest of the code. In general these operators are linear so that the validation of their derivatives is easy. The same code can be used for many test cases, for example using different computation domains and meshes, and the critical point is to affirm that it will be reliable for all the possible test cases (and for all values of the input vector).

The first step is to verify all the chosen methods for different test cases for which we know an analytic solution. This step is necessary but not at all sufficient: for example, for the Darcy equation we know analytic solutions for derivatives for all one-dimensional and pseudo one-dimensional test cases. We do not have simple and general analytical solutions for the other test cases. Onedimensional tests make not possible to verify that we correctly deal with the coupling between the different entries of the hydraulic conductivity tensors.

For test cases without analytic solutions but with a reasonable number of degrees of freedom, we apply also all the implemented differentiation methods. The set of test cases should be representative of all the possible problems, concerning heterogeneity, anisotropy, boundary conditions, but, in order to be exhaustive, for computation time reasons, we use meshes just big enough to make this generality possible. We consider that if all the implemented methods provide the same results for all the test cases it is a very good sign. No small differences can be accepted. Comparison with results of divided differences is not so simple: we must compute divided differences for different differentiation steps, and select the results with "best" differentiation step. This best step is not known before performing a comparison.

For the realistic test case, it can happen that only one method is applicable, regarding memory management and computation time. That is why the previous verification is very important.

We have used divided differences and automatic differentiation with ADOL-C to verify our manual derivated code. The tool ADOL-C seems to be a very safe mean for verification. The tool actually computes both the function and its derivatives. We observed that the only difficulty is to compute the right function. This is verified by comparison of the results provided by ADOL-C and by an original non differentiated code, supposed to be reliable, over a representative set of test cases. Once the right function is computed, the computation of derivatives is always right.

\subsection{Differentiation in direct mode}

In direct mode, the Jacobian matrices are computed column by column by setting elementary variations of the operator $E$ equal to zero. We denote, for $j, j^{\prime} \in\left\{1, \ldots, n_{\text {ip }}\right\},\left(\delta x_{j}\right)_{j^{\prime}}=\delta_{j, j^{\prime}}$ (i.e. 1 when $j=j^{\prime}$ and 0 otherwise). The $j^{\text {th }}$ column of $F^{\prime}$ is

$$
O^{\prime}(\tilde{y}) \delta \tilde{y}^{j}
$$

where $\delta \tilde{y}^{j}$ is defined by

$$
\frac{\partial E}{\partial \tilde{x}} \frac{\partial \tilde{x}}{\partial x} \delta x_{j}+\frac{\partial E}{\partial \tilde{y}} \delta \tilde{y}^{j}=\mathbf{0} .
$$


Thus, independently of the number of output parameters $n_{\mathrm{op}}$, there are $n_{\text {ip }}$ linear systems to solve in order to compute the complete Jacobian matrix of $F$. The size of the linear systems is independent of the number of input or output parameters (for a given state equation $E$ ).

\subsection{Differentiation in reverse mode}

In reverse mode, the Jacobian matrices are computed row by row. Here, we use the adjoint state method which is well suited for the differentiation of implicitly defined functions with a small number of outputs 23$]$.

\subsubsection{The adjoint state method}

The object here is to calculate the $i^{\text {th }}$ row of the Jacobian matrix $F^{\prime}(x)=(O \circ \tilde{F} \circ P)^{\prime}(x)$. The derivation of $P$ is assumed to be very simple. In order to avoid the manipulation of large matrices with $n_{\text {dof }}$ rows, it is better to differentiate directly the composite $O \circ \tilde{F}$ than to differentiate $O$ and $\tilde{F}$ separately. So, we write the Jacobian matrix of the model under the form $F^{\prime}(x)=$ $(O \circ \tilde{F})^{\prime}(\tilde{x}) P^{\prime}(x)$ and we focus on the calculation of the $i^{\text {th }}$ row of $(O \circ \tilde{F})^{\prime}(\tilde{x})$.

Let $G$ be the projection onto the $i^{\text {th }}$ axis in the output space and set $Q=G \circ O$. Then the $i^{\text {th }}$ row of $(O \circ \tilde{F})^{\prime}(\tilde{x})$ is simply the derivative of $g=Q \circ \tilde{F}$, i.e. the transposed of its gradient $\vec{\nabla} g$.

The adjoint state method is popular for minimization problems with equality constraints, and in particular for the computation of the corresponding gradient. Here the gradient $\vec{\nabla} g$ is associated with the function $Q=G \circ O$ and the constraint is the state equation $E(\tilde{x}, \tilde{y})=\mathbf{0}$ defining $\tilde{F}$. Hence, as for minimization problems, we define the following Lagrangian function:

$$
\begin{aligned}
\mathcal{L}: \mathcal{P} \times \mathbb{R}^{n_{\text {dof }}} \times \mathbb{R}^{n_{\text {dof }}} & \rightarrow \mathbb{R}, \\
(\tilde{x} ; \tilde{y}, \lambda) & \mapsto Q(\tilde{y})+\langle E(\tilde{x}, \tilde{y}), \lambda\rangle,
\end{aligned}
$$

where $\lambda \in \mathbb{R}^{n_{\text {dof }}}$ is the Lagrange multiplier associated with the constraint (here the size of the state equation is the size of $\tilde{y}$, i.e. $\left.n_{\text {dof }}\right)$. Then, for a given parameter $\tilde{x}$, let $\tilde{y}_{\tilde{x}}$ denote the state variable solution of the equation $E(\tilde{x}, \tilde{y})=\mathbf{0}$, i.e. $\tilde{y}_{\tilde{x}}=\tilde{F}(\tilde{x})$. The state variable $\tilde{y}_{\tilde{x}}$ is also characterized by the equation

$$
\forall \delta \lambda \in \mathbb{R}^{n_{\mathrm{dof}}}, \quad \frac{\partial \mathcal{L}}{\partial \lambda}\left(\tilde{x} ; \tilde{y}_{\tilde{x}}, \lambda\right) \delta \lambda=\mathbf{0} .
$$

This condition is independent of the variable $\lambda$. Similarly, if $Q$ and $E$ are sufficiently regular, the adjoint state variable $\lambda_{\tilde{x}}$ may be defined by the equation

$$
\forall \delta \tilde{y} \in \mathbb{R}^{n_{\mathrm{dof}}}, \quad \frac{\partial \mathcal{L}}{\partial \tilde{y}}\left(\tilde{x} ; \tilde{y}_{\tilde{x}}, \lambda_{\tilde{x}}\right) \delta \tilde{y}=\mathbf{0},
$$

and $\lambda_{\tilde{x}}$ is the solution of the linear equation

$$
\left[\frac{\partial E}{\partial \tilde{y}}\left(\tilde{x}, \tilde{y}_{\tilde{x}}\right)\right]^{T} \lambda_{\tilde{x}}=-\left[Q^{\prime}\left(\tilde{y}_{\tilde{x}}\right)\right]^{T} .
$$

Moreover we have $\forall \tilde{x} \in \mathcal{P}, \quad g(\tilde{x})=\mathcal{L}\left(\tilde{x} ; \tilde{y}_{\tilde{x}}, \lambda_{\tilde{x}}\right)$ because $\tilde{y}_{\tilde{x}}$ satisfies $E\left(\tilde{x}, \tilde{y}_{\tilde{x}}\right)=\mathbf{0}$. Hence, if $E$ is sufficiently regular,

$$
\begin{aligned}
\langle\vec{\nabla} g, \delta \tilde{x}\rangle & =\frac{\partial \mathcal{L}}{\partial \tilde{x}}\left(\tilde{x} ; \tilde{y}_{\tilde{x}}, \lambda_{\tilde{x}}\right) \delta \tilde{x}+\frac{\partial \mathcal{L}}{\partial \tilde{y}}\left(\tilde{x} ; \tilde{y}_{\tilde{x}}, \lambda_{\tilde{x}}\right) \frac{\partial \tilde{y}_{\tilde{x}}}{\partial \tilde{x}} \delta \tilde{x}+\frac{\partial \mathcal{L}}{\partial \lambda}\left(\tilde{x} ; \tilde{y}_{\tilde{x}}, \lambda_{\tilde{x}}\right) \frac{\partial \lambda_{\tilde{x}}}{\partial \tilde{x}} \delta \tilde{x} \\
& =\frac{\partial \mathcal{L}}{\partial \tilde{x}}\left(\tilde{x} ; \tilde{y}_{\tilde{x}}, \lambda_{\tilde{x}}\right) \delta \tilde{x} .
\end{aligned}
$$

If $\tilde{F}$ and $Q$ are sufficiently regular then $g$ is differentiable and its gradient is

$$
\vec{\nabla} g=\left[\frac{\partial E}{\partial \tilde{x}}\left(\tilde{x}, \tilde{y}_{\tilde{x}}\right)\right]^{T} \lambda_{\tilde{x}}
$$




\subsubsection{Assembling the Jacobian matrix row by row}

Equations (55) and (6) are now used to compute the $i^{\text {th }}$ row of $F^{\prime}$.

Let $g_{\tilde{y}, i}$ denote the $i^{\text {th }}$ row of the Jacobian matrix of the measure operator $O$ :

$$
g_{\tilde{y}, i}=G \circ O^{\prime}(\tilde{y}) .
$$

According to equation (5), the corresponding adjoint state $\lambda^{i}$ is determined by solving

$$
\left[\frac{\partial E}{\partial \tilde{y}}\left(\tilde{x}, \tilde{y}_{\tilde{x}}\right)\right]^{T} \lambda^{i}=-g_{\tilde{y}, i}^{T} .
$$

Then, according to equation (6), the $i^{\text {th }}$ row of the Jacobian matrix of $F$ is

$$
g_{x, i}=\lambda^{i^{T}}\left[\frac{\partial E}{\partial \tilde{x}} \frac{\partial \tilde{x}}{\partial x}\right] .
$$

So there is one linear system to solve for each output parameter in order to compute the complete Jacobian matrix of $F$. The number of linear systems to be solved is independent of the length of the vector $x$. The size of the systems to be solved is the same as for direct mode. 


\section{Mathematical and Numerical Models}

\subsection{The Steady-state Darcy flow problem}

Assume that the calculation domain, identified with the porous medium containing the nuclear waste storage site, is a bounded open subdomain $\Omega$ of $\mathbb{R}^{3}$. Assume that the medium is saturated with water, which is supposed to be incompressible. Assume further that the shape of the domain is such that the effects of gravity are negligible. Then flow in $\Omega$ is governed by a stationary conservation law together with Darcy's law:

$$
\begin{aligned}
& \operatorname{div} \vec{u}=q \quad \text { in } \Omega \quad \text { (conservation law) } \\
& \vec{u}=-\mathcal{K} \vec{\nabla} p \quad \text { in } \Omega \quad(\text { Darcy's law }),^{\prime}
\end{aligned}
$$

where $\vec{u}$ is the Darcy water flow velocity field, $q$ is a source term, $\mathcal{K}$ is the non homogeneous hydraulic conductivity matrix field, which is assumed to be everywhere symmetric and positive definite, and $p$ is the pressure field. Assume that the boundary of $\Omega$, is divided into two parts, $\Gamma_{D}$ and $\Gamma_{N}$, on which are imposed Dirichlet and Neumann boundary conditions respectively:

$$
\begin{aligned}
& p=p_{D} \text { on } \Gamma_{D} \\
& \vec{u} \cdot \vec{n}=g_{N} \text { on } \Gamma_{N} \text {, }
\end{aligned}
$$

where $\partial \Omega=\bar{\Gamma}_{D} \cup \bar{\Gamma}_{N}$ with $\Gamma_{D} \cap \Gamma_{N}=\emptyset$.

This work is concerned with the effect of uncertainties about the hydraulic conductivity matrix field $\mathcal{K}$ on certain safety indicators. Here the safety indicators are taken to be the fluxes of $\vec{u}$ through $n_{\mathrm{op}}$ specified surfaces in $\bar{\Omega}$ called outlet channels $S_{i}, i=1, \ldots, n_{\mathrm{op}}$. Thus given these $n_{\mathrm{op}}$ outlet channels, oriented by the unitary normal vector fields $\vec{n}^{i}, i=1, \ldots, n_{\mathrm{op}}$, the safety indicators are the fluxes $\Phi_{i}, i=1, \ldots, n_{\mathrm{op}}$ defined by

$$
\text { for } i=1, \ldots, n_{\mathrm{op}}, \quad \Phi_{i}=\int_{S_{i}} \vec{u} \cdot \vec{n}^{i} .
$$

The hydraulic conductivity matrix field is assumed to be piecewise constant so that $\bar{\Omega}$ is the union of the closures of $n_{z}$ mutually exclusive subdomains $\Omega_{\alpha}, \alpha=1, \cdots, n_{z}$ on which $\mathcal{K}$ is constant:

$$
\mathcal{K}_{\mid \Omega_{\alpha}} \equiv \mathbf{K}_{\alpha}
$$

where for each $\alpha, \alpha=1, \cdots, n_{z}, \mathbf{K}_{\alpha}$ is a symmetric $3 \times 3$ matrix. Thus $\mathcal{K}$ is uniquely determined by $6 n_{z}$ parameters though the number of parameters maybe reduced in the presence of further assumptions concerning the form of the matrices $K_{\alpha}$ or assumptions that in certain zones $K_{\alpha}$ is well known.

For the problem treated here, it is supposed that, for each zone $\alpha, \mathbf{K}_{\alpha}$ is either diagonal or scalar, so that the total number $n_{\text {ip }}$ of independent input parameters is less than or equal to $3 n_{z}$. Let $K=\left(K_{j}\right)_{j=1, \ldots, n_{\text {ip }}}$ denote the vector containing these parameters. As usual with elliptic equations, and since the values of the hydraulic conductivity often vary over several orders of magnitude, it is reasonable to use a logarithmic parametrization; i. e. to take as input parameters the logarithms of the values $K_{j}$ :

$$
\text { for } j=1, \ldots, n_{\mathrm{ip}}, \quad \text { let } x_{j}=\log K_{j},
$$

and let $x=\left(x_{j}\right)_{j=1, \ldots, n_{\mathrm{ip}}}$.

The function we are interested in is the function $\mathcal{F}$ that associates output parameters $\Phi=$ $\left(\Phi_{i}\right)_{i=1, \ldots, n_{\mathrm{op}}}$ to input parameters $x=\left(x_{j}\right)_{j=1, \ldots, n_{\mathrm{ip}}}$, a function from $\mathbb{R}^{n_{\mathrm{ip}}}$ to $\mathbb{R}^{n_{\mathrm{op}}}$. But, as we are dealing with numerical calculations, a discretization $F$ of this function needs to be defined. The function $F$ will associate approximations $y=\left(y_{i}\right)_{i=1, \ldots, n_{\mathrm{op}}}$ of the fluxes $\Phi=\left(\Phi_{i}\right)_{i=1, \ldots, n_{\mathrm{op}}}$ through the outlet channels $\left(S_{i}\right)_{i=1, \ldots, n_{\mathrm{op}}}$ to the input parameters $x=\left(x_{j}\right)_{j=1, \ldots, n_{\mathrm{ip}}}$. 


\subsection{Discretization}

To obtain the discretization $F$ of the function $\mathcal{F}$ a mixed-hybrid finite element method [31] is used. Assume that $\mathcal{T}_{h}=\left(T_{i}\right)_{i \in I}$ is a finite element discretization of the domain $\Omega$, that is a "triangulation" of $\Omega$ which may be made up of tetrahedra, or of convex hexahedral elements. The mesh $\mathcal{T}_{h}$ is such that the outlet channels $S_{i}, i=1, \ldots, n_{\mathrm{op}}$, are unions of faces of the mesh. The set of faces of the elements of the triangulation $\mathcal{T}_{h}$ (including those faces contained in the interior of $\Omega$ and those lying on the boundary of $\Omega$ ) is denoted $\mathcal{S}_{h}=\left(E_{j}\right)_{j \in J}$. Each face $E_{j}$ is oriented by an arbitrarily chosen unit normal vector $\vec{n}_{j}$. In the case of tetrahedral or parallelepiped cells, the lowest order Raviart-Thomas-Nedelec elements [30, 28, are used, and in the case of more general hexahedral cells, the composite elements defined in [33, 34] are used. In each case, the degrees of freedom for such a method are of three types:

- The pressure unknowns: for each cell $T_{i}$, one value corresponding to an approximation to the average value of the pressure on the cell.

- The trace-of-pressure unknowns: for each face $E_{j}$ not on the Dirichlet boundary $\Gamma_{D}$, one value corresponding to an approximation of the average value of the pressure on the face.

- The flux unknowns: for each cell $T_{i}$ and for each face $E_{j}$ of $T_{i}$, one value corresponding to an approximation of the outgoing flux from $T_{i}$ through $E_{j}$. Thus we have one flux value per face of the boundary, and two flux values per interior face. The continuity of the flow through the interior faces is imposed not by the choice of the unknowns but through supplementary equations, obtained by using Lagrange multipliers associated with the traceof-pressure unknowns for the interior faces through the operator $C$ of equation (13) below. Similarly the Neumann boundary conditions are imposed by using Lagrange multipliers associated with the trace-of-pressure unknowns for the faces on the Neumann boundary $\Gamma_{N}$.

The vector of unknowns will be denoted $Y$ :

$$
Y=\left(\begin{array}{c}
U \\
P \\
L
\end{array}\right),
$$

where the components of $U$ are approximations to the fluxes of $\vec{u}$ through the faces of the mesh $\left(E_{j}\right)_{j \in J}$ (one component $U_{j}$ for each boundary face and two components $U_{j}$ and $U_{j^{\prime}}$ for each interior face: $U_{j} \approx \int_{E_{j}} \vec{u} \cdot \overrightarrow{\mathrm{n}}_{j}$ and $\left.U_{j^{\prime}} \approx \int_{E_{j}} \vec{u} \cdot\left(-\overrightarrow{\mathrm{n}}_{j}\right)\right), P$ contains one pressure value per cell and $L$ contains the pressure traces, one per face not included in $\Gamma_{D}$.

The discretized variational formulation then yields the following algebraic system

$$
\mathcal{E}_{h}(\mathcal{K}, Y)=\mathbf{0},
$$

with

$$
\mathcal{E}_{h}(\mathcal{K}, Y)=M(\mathcal{K}) Y-\left(\begin{array}{c}
R_{1} \\
R_{2} \\
R_{3}
\end{array}\right),
$$

where $M(\mathcal{K})$ is a symmetric positive definite matrix of the form

$$
M(\mathcal{K})=\left(\begin{array}{ccc}
A\left(\mathcal{K}^{-1}\right) & { }^{t} B & { }^{t} C \\
B & \mathbf{0} & \mathbf{0} \\
C & \mathbf{0} & \mathbf{0}
\end{array}\right) .
$$

The first equation $A\left(\mathcal{K}^{-1}\right) U+{ }^{t} B P+{ }^{t} C L=R_{1}$ corresponds to Darcy's law where $R_{1}$ depends on the Dirichlet boundary conditions. The second equation $B U=R_{2}$ corresponds to the conservation law where $R_{2}$ contains the source term. The third equation $C U=R_{3}$ expresses the continuity of the velocity flux where $R_{3}$ contains Neumann boundary conditions. 
The vectors of real numbers $Y$ and $\mathcal{E}_{h}(\mathcal{K}, Y)$ are of size $n_{\mathrm{dof}}$, where $n_{\mathrm{dof}}$ is the number of degrees of freedom.

Then the function to be analyzed is

$$
F=\left(\begin{array}{c}
F_{1} \\
\ldots \\
F_{n_{\mathrm{op}}}
\end{array}\right)
$$

with, for $i=1, \ldots, n_{\mathrm{op}}$,

$$
\begin{aligned}
F_{i}: \mathbb{R}^{n_{\mathrm{ip}}} & \rightarrow \mathbb{R}, \\
x & \mapsto y_{i}=\sum_{j \in J \mid E_{j} \subset S_{i}} \overrightarrow{\mathrm{n}}_{j} \cdot \vec{n}^{i} U_{j}
\end{aligned}
$$

where $\vec{n}_{j} \cdot \vec{n}^{i}= \pm 1$ depending on whether $\vec{n}_{j}=\vec{n}^{i}$ or $\vec{n}_{j}=-\vec{n}^{i}$. The function $F$ is decomposed as a composite of a parametrization operator, a fine model operator and an observation operator: $F=O \circ \tilde{F} \circ P$, see Section 3.1 The parametrization operator $P$ is defined by

$$
P: x \in \mathbb{R}^{n_{\text {ip }}} \mapsto \mathcal{K} \in \mathcal{P}
$$

where the space $\mathcal{P}$ for fine parameters is the space of hydraulic conductivity fields

$$
\mathcal{P}=\left\{\mathcal{K}: \Omega \rightarrow \mathcal{M}_{3 \times 3}^{+} \mid \mathcal{K}\right. \text { has bounded and measurable coefficients }
$$

and is uniformly elliptic

with $\mathcal{M}_{3 \times 3}^{+}$the set of symmetric positive definite matrices of size $3 \times 3$. The fine model $\tilde{F}$ is implicitly defined by the state equation $Y=\tilde{F}(\mathcal{K}) \Leftrightarrow \mathcal{E}_{h}(\mathcal{K}, Y)=\mathbf{0}$ where $n_{\text {op }}$ is the number of outlet channels and the observation or measure operator is

$$
O: Y \in \mathbb{R}^{n_{\mathrm{dof}}} \mapsto y \in \mathbb{R}^{n_{\mathrm{op}}}
$$

Remark 1 It is important to note that in the method proposed, the matrix $M(\mathcal{K})$ of equations (12) and (13) will never be constructed and a system of size $n_{\mathrm{dof}} \times n_{\mathrm{dof}}$ will never be solved. Instead, an equation in the unknown vector $L$, obtained by eliminating the variables $U$ and $P$, is solved. As $A\left(\mathcal{K}^{-1}\right), B$ and $C$ are block matrices, with small blocks, $U$ and $P$ can be eliminated by inverting only local matrices. (For $A\left(\mathcal{K}^{-1}\right)$, the blocks are of size $6 \times 6$ in the case of hexahedral meshes or of size $4 \times 4$ in the case of tetrahedral meshes, one block corresponding to one element of $\mathcal{T}_{h}$.) Once $L$ has been calculated, $P$ and $U$ can be found by local matrix multiplications. See for example 47.

\subsection{Differentiation}

\subsubsection{Direct mode}

This section concerns the construction and resolution of equation (4).

The vector $\delta Y^{j}$ is actually the solution of a boundary value problem of the same form as that for $Y$ (12):

$$
\mathrm{d} \mathcal{E}_{h_{\mathcal{K}, \mathrm{d} \mathcal{K}}}\left(\delta Y^{j}\right)=\mathbf{0},
$$

where

$$
\begin{gathered}
\delta Y^{j}=\left(\begin{array}{c}
\delta U^{j} \\
\delta P^{j} \\
\delta L^{j}
\end{array}\right) ; \\
\mathrm{d} \mathcal{E}_{h_{\mathcal{K}, \delta \mathcal{K}}}\left(\delta Y^{j}\right)=M(\mathcal{K}) \delta Y^{j}-\left(\begin{array}{c}
A\left(\mathcal{K}^{-1} \delta \mathcal{K} \mathcal{K}^{-1}\right) U \\
\mathbf{0} \\
\mathbf{0}
\end{array}\right),
\end{gathered}
$$


with $\delta \mathcal{K}=\frac{\partial \mathcal{K}}{\partial x_{j}} \delta x_{j}$ and with $U$ defined as in equation (11), the function $A$ which takes as input a field of $3 \times 3$ symmetric matrices and returns a square matrix of the same size as $U$ is the same as the one used in equation (13) and $M(\mathcal{K})$ is defined as in equation (13). Remark 10 is pertinent here.

\subsubsection{Reverse mode}

The expression of $g_{\tilde{y}, i}$ defined in equation (17) is for our example $(\tilde{y}=Y)$ very simple: for $i=$ $1, \ldots, n_{\mathrm{op}}, g_{Y, i}^{T}$ is the vector of the same length as $Y$ whose $k^{t h}$ component is equal to $\overrightarrow{\mathrm{n}}_{k} \cdot \vec{n}^{i}$ if $E_{k}$ exists with $E_{k} \subset S_{i}$ and equal to 0 otherwise.

It is easy to see what equation (8) is in the present context since the partial function to be differentiated is linear.

The remainder of this section concerns the calculation of the right hand side of equation (9). For $j \in I$, we denote $U^{j}$ the vector of size $n_{j}$ containing the components of $U$ representing the outgoing flow from $T_{j}$. The contribution of the cell $T_{j}$ to the global mass matrix $A\left(\mathcal{K}^{-1}\right)$ is represented by the elementary matrix $A_{j}\left(\mathcal{K}^{-1}\right)$. It is a square matrix of size $n_{j} \times n_{j}$, with $n_{j}$ the number of edges of $T_{j}$. For $i, j \in I, \lambda^{i, j}$ is the vector containing the components of $\lambda^{i}$ associated with faces of $T_{j}$.

For $k, l=1, \ldots, 3$, define the field of $3 \times 3$ matrices $\mathcal{H}^{(k, l)}$ by

$$
\text { for } p, q=1, \ldots, 3, \mathcal{H}_{p, q}^{(k, l)}=-\left(\mathcal{K}^{-1}\right)_{p, k}\left(\mathcal{K}^{-1}\right)_{l, q} .
$$

Recall that $\mathcal{K}$ is supposed to be piecewise constant; see equation (10). For $\alpha=1, \ldots, n_{z}$ and for $k=1, \ldots, 3$ we have

$$
\frac{\partial y_{i}}{\partial \mathbf{K}_{\alpha_{k, k}}}=\sum_{j \in I \mid T_{j} \subset \Omega_{\alpha}}{ }^{t} U^{j} A_{j}\left(\mathcal{H}^{(k, k)}\right) \lambda^{i, j} .
$$

As the matrices $\mathbf{K}_{\alpha}$ for $\alpha=1, \ldots, n_{z}$ have been supposed to be symmetric it follows that, for $\alpha=1, \ldots, n_{z}, k=1, \ldots, 3$ and $l=1, \ldots, k-1$, the matrix $\left(\mathcal{H}^{(k, l)}+\mathcal{H}^{(l, k)}\right)$ is symmetric and we have

$$
\frac{\partial y_{i}}{\partial \mathbf{K}_{\alpha_{k, l}}}=\sum_{j \in I \mid T_{j} \subset \Omega_{\alpha}}{ }^{t} U^{j} A_{j}\left(\mathcal{H}^{(k, l)}+\mathcal{H}^{(l, k)}\right) \lambda^{i, j} .
$$




\section{$5 \quad$ Numerical results}

We have applied the Monte Carlo method and the deterministic approach for sensitivity analysis to a realistic problem of flow in porous media. After a description of the parameters of the test case we give results and conclusions obtained with both approaches to compare them.

\subsection{Test case description}

The test case that we consider models water flow around a potential underground nuclear waste storage site. The data for this test case were provided by ANDRA, see [27].

\subsubsection{The computational domain}

The model is a simplified 3-dimensional hydraulic model, mostly made up of plane parallel layers. The computational domain is a layered medium $\Omega$, roughly 500 meters deep and of a horizontal extent of about 40 kilometers by 40 kilometers. It is divided into 12 zones $\left(\Omega_{\alpha}\right), \alpha=1, \ldots, 12$, each zone being contained in a single layer. A computational mesh of roughly 300,000 cells, each cell being contained in one zone, is used. The zones are represented in the two dimensional sections shown in Figure 10 The storage site is contained in zone $\Omega_{1}$ and is shown in red in the figure. In all of the figures vertical dimensions have been multiplied by a factor of 30 . The hydraulic
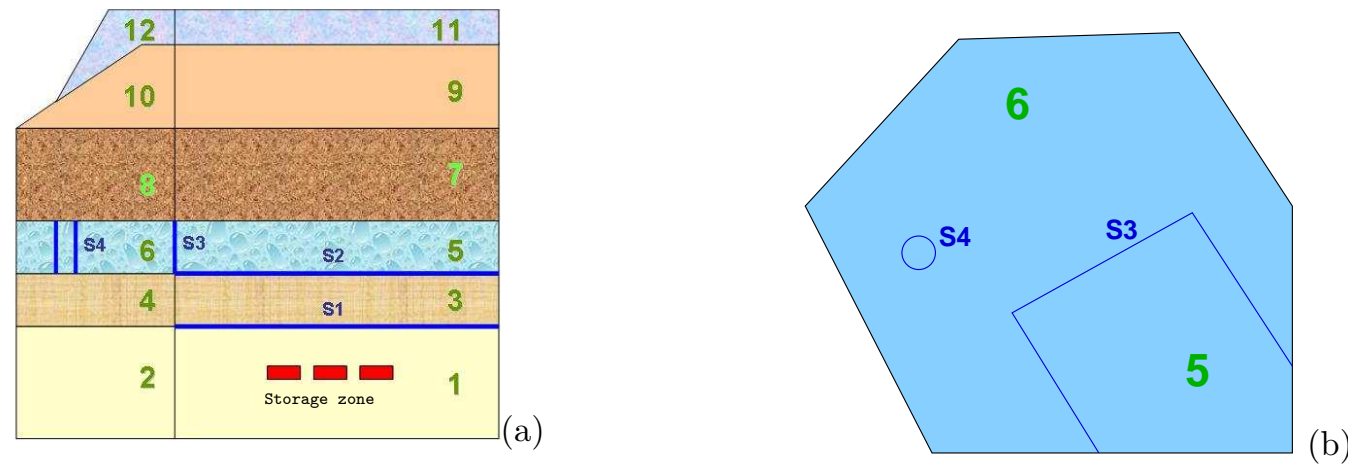

Figure 1: 2D vertical section $(a)$ and horizontal section $(b)$ of the 3D hydraulic model. S1, .., S4 are the outlet channels. Green numbers are the indices for zones. $\mathbf{K}_{1}=\mathbf{K}_{2}=\operatorname{diag}\left(k_{h}, k_{h}, k_{v}\right)$, $\mathbf{K}_{i}=k_{i} \mathbf{I}$ for $i=3, \ldots, 12 . k_{h}, k_{v}$ and $k_{i}, i=3, \ldots, 6$ are uncertain.

conductivity is constant in each zone and the formalism described in sections 4.1 and 4.2 is used. The hydraulic conductivity matrices in zones $\Omega_{1}$ and $\Omega_{2}$ are the same, $\mathbf{K}_{1}=\mathbf{K}_{2}$. This matrix is diagonal with a vertical component $k_{v}$ and with both horizontal components equal to $k_{h}$. In zones $\Omega_{3}$ through $\Omega_{12}$ the hydraulic conductivity is scalar: for $\alpha=3, \ldots, 12, \mathbf{K}_{\alpha}=k_{\alpha} \mathbf{I}$, where $\mathbf{I}$ is the $3 \times 3$ identity matrix. The vector $K$ is of length $n_{\text {ip }}=12$ with $K_{1}=k_{h}, K_{2}=k_{v}$ and for $\alpha=3, \ldots, 12 K_{\alpha}=k_{\alpha}$. In the figures and tables that follow, the first two components are indexed by $h$ and $v$, instead of by 1 and 2 .

There are 4 outlet channels $\left(S_{i}\right)_{i=1, \ldots, 4}$, which are also shown in dark blue in Figure 1 The outlet channels $S_{1}$ and $S_{2}$ are horizontal plane surfaces coinciding with interfaces between zones: $S_{1}=\bar{\Omega}_{1} \cap \bar{\Omega}_{3}$ and $S_{2}=\bar{\Omega}_{3} \cap \bar{\Omega}_{5}$. The outlet channel $S_{3}$ is made up of 3 vertical plane surfaces with $S_{3}=\bar{\Omega}_{5} \cap \bar{\Omega}_{6}$. The outlet channel $S_{4}$ is the lateral face of a cylinder with a vertical axis, with $S_{4} \subset \Omega_{6}$.

Figure 2 shows from two different perspectives the pressure distribution corresponding to the most likely set of hydraulic conductivities (corresponding to maximum of PDF), whose values are given in Table 2 and 3 Water globally flows from regions of higher pressure (shown in red) to regions of lower pressure (shown in blue). Figure 3 shows for the same hydraulic conductivities 
and from the same perspectives a single stream line emanating from the storage site. It goes through the outlet channels $S_{1}, S_{2}, S_{3}$ and along the axis of the cylinder defining $S_{4}$.
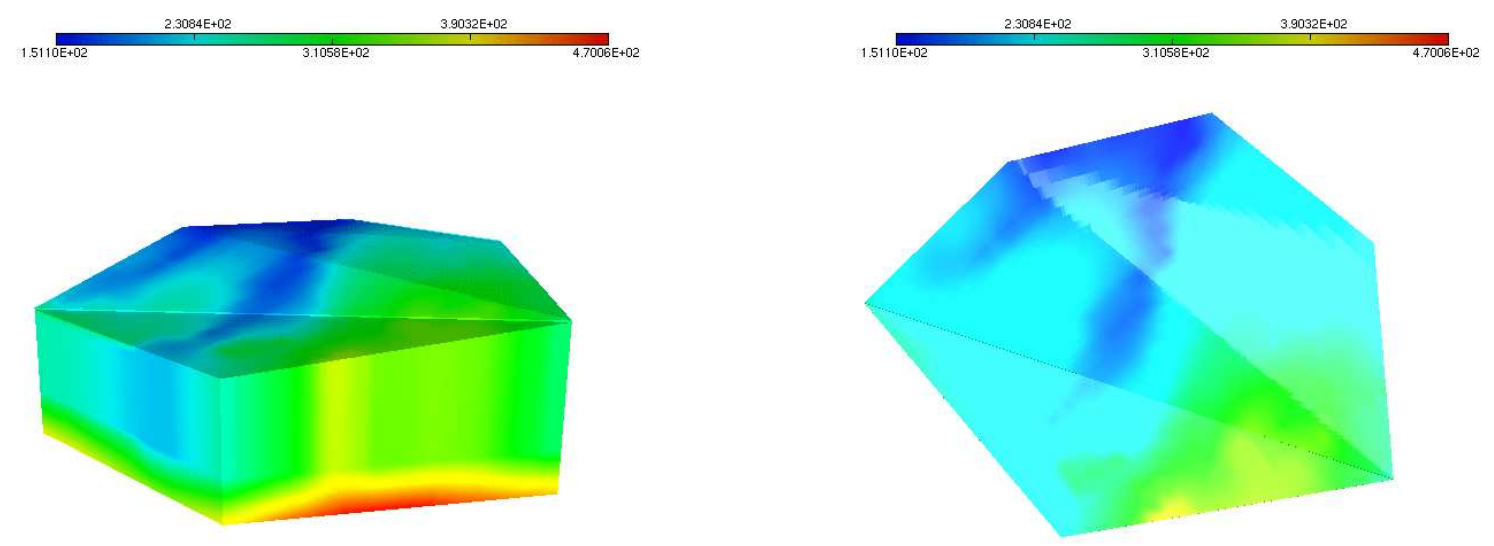

(a)

Figure 2: The pressure field for the most likely set of parameters. (a): front view; $(b)$ : top view.
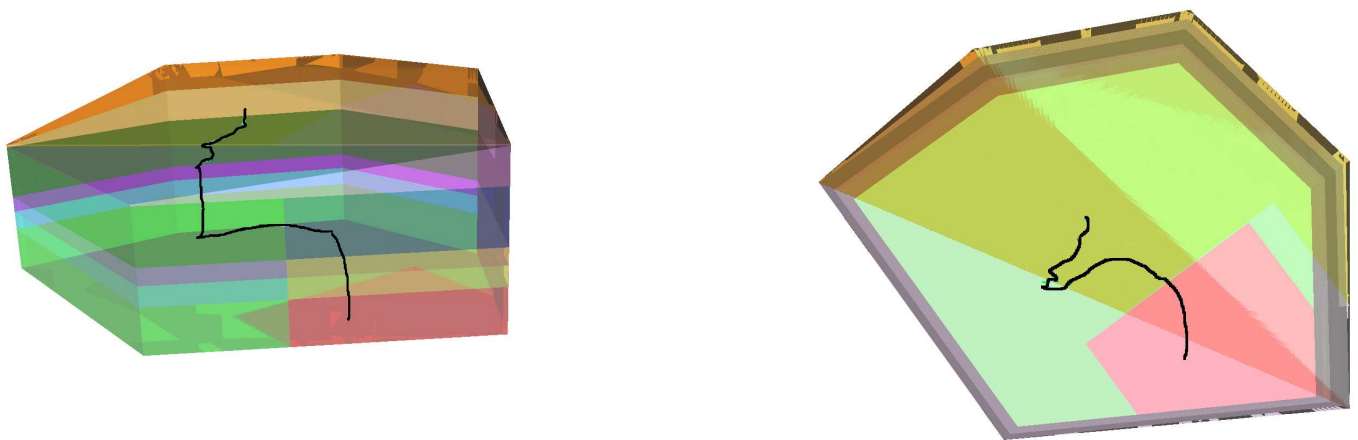

(a)

Figure 3: A water velocity stream line for the most likely set of parameters. $(a)$ : front view; $(b)$ : top view. The computation domain is transparent and different background colors correspond to different zones.

\subsubsection{Data for input parameters}

It is assumed that the hydraulic conductivities in zones 7 through 12 are precisely known and their values, $k_{7}, \ldots, k_{12}$, are given in Table 2 The other variables, $k_{h}, k_{v}, k_{3}, \ldots, k_{6}$, are defined by the probability laws given in Table 1 we see that some of them are not independent (e.g. $k_{h}$ and $k_{v}$ or $k_{3}$ and $k_{4}$ ). The values for these parameters considered as the most likely are given in Table 3 The choice of logarithmic parametrization for the deterministic study is coherent with the description of probabilistic distributions by log-normal laws.

\subsubsection{Expected sensitivity results}

The mean flow is largely determined by the values of the hydraulic conductivity in the less permeable layers, which behave like barriers, and by the boundary conditions, whose influence is not studied here (see the importance of the boundary conditions in [22]). See also the results for both approaches applied to the one-dimensional case in section 2.3 The heterogeneities of the 


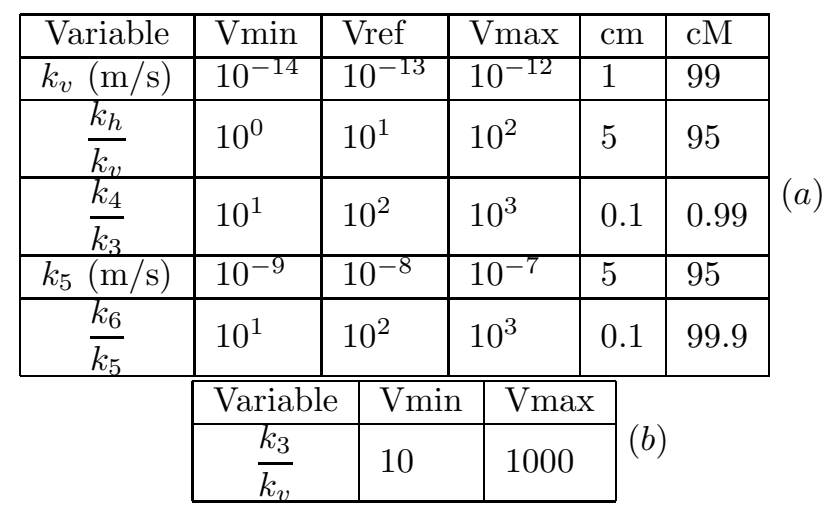

Table 1: Basis variables, and parameters for the PDF used to generate the samples of input parameters. The variables in table $(a)$ follow log-normal laws truncated at the centiles $\mathrm{cm}$ and cM, of mean Vref and of standard deviation such that the law loads the interval [Vmin,Vmax]; the variable in table $(b)$ follows an uniform law of minimum Vmin and of maximum Vmax.

\begin{tabular}{|c|c|c|c|c|c|}
\hline$k_{7}$ & $k_{8}$ & $k_{9}$ & $k_{10}$ & $k_{11}$ & $k_{12}$ \\
\hline $10^{-9}$ & $210^{-7}$ & $10^{-12}$ & $10^{-11}$ & $310^{-4}$ & $310^{-5}$ \\
\hline
\end{tabular}

Table 2: Invariant hydraulic conductivities $(\mathrm{m} / \mathrm{s})$.

\begin{tabular}{|}
\begin{tabular}{|c|c|c|c|c|c|}
\hline$k_{h}$ & $k_{v}$ & $k_{3}$ & $k_{4}$ & $k_{5}$ & $k_{6}$ \\
\hline $10^{-11}$ & $10^{-13}$ & $10^{-11}$ & $10^{-9}$ & $810^{-9}$ & $610^{-7}$ \\
\hline \multicolumn{2}{|c|}{$y_{1}$} & $y_{2}$ & \multicolumn{2}{|c|}{$y_{3}$} & $y_{4}$ \\
\hline $2.4810^{-5}$ & $2.4610^{-5}$ & 1.44 & $10^{-4}$ & $5.5110^{-3}$ \\
\hline
\end{tabular}
\end{tabular}

Table 3: The values considered as most likely for the variable input parameters $(\mathrm{m} / \mathrm{s})$ and corresponding water fluxes through the outlet channels $\left(\mathrm{m}^{3} / \mathrm{s}\right)$.

hydraulic conductivity have an influence on the spatial variation of the flow: the water flux is indeed attracted toward the more permeable zones, which behave like sponges.

\subsection{Results of the probabilistic study}

Monte Carlo method was used. We needed to perform $N=1000$ simulations for this probabilistic study.

The sensitivity of $y_{2}$ has not been studied since it was observed that $y_{2}$ is always very close to $y_{1}$. The correlation indicator for the results presented is the mean of the coefficients of Spearman, the PRCC and the SRRC. Results are given in Table 4 we see that $y_{1}$ depends exclusively on $k_{v}$, $y_{3}$ depends largely on $k_{5}$ and to a lesser extent on $k_{6}$ and $y_{4}$ depends exclusively on $k_{6}$.

These results are not surprising e.g. with respect to section [5.1.3 For example the mean vectical flux is governed by the vertical hydraulic conductivity in the less permeable zone and horizontal fluxes are governed by upwind permeabilities.

\subsection{Deterministic results}

The Jacobian matrix of the problem has been computed using the adjoint state method since outputs are less numerous than inputs, and using a manually exactly differentiated code. 


\begin{tabular}{|c|c|c|c|c|c|}
\hline$y_{1}$ & indicator & $y_{3}$ & indicator & $y_{4}$ & indicator \\
\hline$\left(k_{h}\right)$ & 0.10 & $\left(k_{h}\right)$ & 0.03 & $\left(k_{h}\right)$ & 0.01 \\
$\left(\mathbf{k}_{\mathbf{v}}\right)$ & $\mathbf{1 . 0 0}$ & $\left(k_{v}\right)$ & 0.05 & $\left(k_{v}\right)$ & 0.01 \\
$\left(k_{3}\right)$ & 0.08 & $\left(k_{3}\right)$ & 0.00 & $\left(k_{3}\right)$ & 0.03 \\
$\left(k_{4}\right)$ & 0.02 & $\left(k_{4}\right)$ & 0.01 & $\left(k_{4}\right)$ & 0.02 \\
$\left(k_{5}\right)$ & 0.12 & $\left(\mathbf{k}_{\mathbf{5}}\right)$ & $\mathbf{0 . 9 3}$ & $\left(k_{5}\right)$ & 0.14 \\
$\left(k_{6}\right)$ & 0.08 & $\left(\mathbf{k}_{\mathbf{6}}\right)$ & $\mathbf{0 . 4 7}$ & $\left(\mathbf{k}_{\mathbf{6}}\right)$ & $\mathbf{1 . 0 0}$ \\
\hline
\end{tabular}

Table 4: Statistical indicator of correlation between the water flows and the input parameters.

\subsubsection{Interpretation of the SVD results}

Each set of three pictures from Figure 4 to Figure 8 should be read as follows.

- The first picture $(a)$ gives the singular values, normalized with respect to the first singular value. The coefficients of normalization are given in square brackets. We have $n_{o c}=4$ output parameters (one for each outlet channel) and $n_{o c}<n_{\text {ip }}\left(n_{\text {ip }}\right.$ is the number of input parameters), so we have 4 singular values $\left\{s_{k}\right\}_{k=1, \ldots, 4}$, each represented by one colored bullet in picture $(a)$.

- The second picture $(b)$ gives the first 4 singular vectors of the input space represented in the canonical basis corresponding to the components of $K$. We have $n_{\text {ip }}=12$ input parameters so 12 input singular vectors $\left\{v_{k}\right\}_{k=1, \ldots, 12}$. Each of the 4 curves corresponds to one singular vector and each of the 4 curves is associated with the singular value of the same color in the first picture. For $k>n_{o c}=4$, the singular vector of the input space $v_{k}$ has no influence on the outputs and is not represented in the picture. Such a vector is in the kernel of the Jacobian matrix.

- The third picture $(c)$ gives the singular vectors of the output space represented in the canonical basis corresponding to the components of $y$. We have 4 output parameters so 4 output singular vectors $\left\{u_{k}\right\}_{k=1, \ldots, 4}$. Each curve corresponds to one singular vector and is associated with the singular value of the same color in the first picture and with the singular vector of the same color in the second picture through equation (2).

We use equation (3) to interpret the results: for example, if the $i^{\text {th }}$ components of $v_{k}, s_{k}$ and the $j^{t h}$ component of $u_{j}$ are nonzero then the variation of $y_{j}$ depends in particular on the $k_{i}$. On the other hand, if the $i^{t h}$ component of $v_{k}$ is equal to zero, then the variation of the linear combination of fluxes $\left\langle u_{k}, y\right\rangle$ is locally independent of the variation of $k_{i}$. For each singular value, one has to consider the correspondingly colored line in the middle figure to check for the influencing parameters, and in the right figure to check for the affected fluxes.

\subsubsection{A first local study}

The results shown in Figure 4 are obtained with the most likely parameters, given in Tables 2 and [3]

Around this set of parameters, the main influence is that of the hydraulic conductivity in zone number 6 on the flux $y_{4}$ through the surface $S_{4}$. This influence is indeed represented by the red bullet on figure $4(a)$ and the red curves on figures $4(b)$ and $4 .(c)$. The singular vector $v_{1}$ on figure $4(b)$ has exactly 1 nonzero component, its $6^{\text {th }}$ component, corresponding to hydraulic conductivity in zone number 6 , and the unique nonzero component of the corresponding singular vector $u_{1}$ represented on figure 4 (c) is its $4^{\text {th }}$ component.

Next we have the influence of the hydraulic conductivity in zone number 5 on $y_{3}$. It is represented in dark blue on the figures. The only nonzero component of $v_{2}$ on figure 4 ( $(b)$ is its $5^{t h}$ component and the only nonzero component of $u_{2}$ on figure $4 .(c)$ is its $3^{\text {rd }}$ component. 


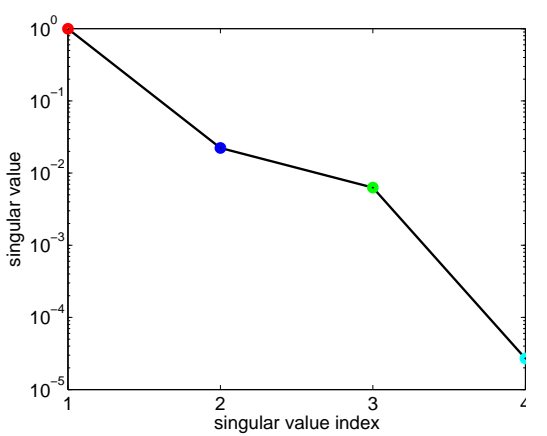

(a) $\left[5.5310^{-3} \mathrm{~m}^{3} / \mathrm{s}\right]$

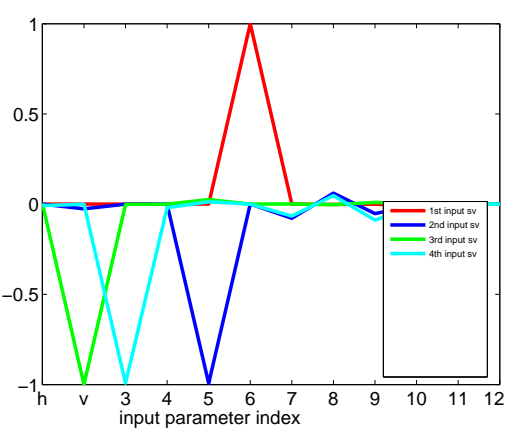

(b)

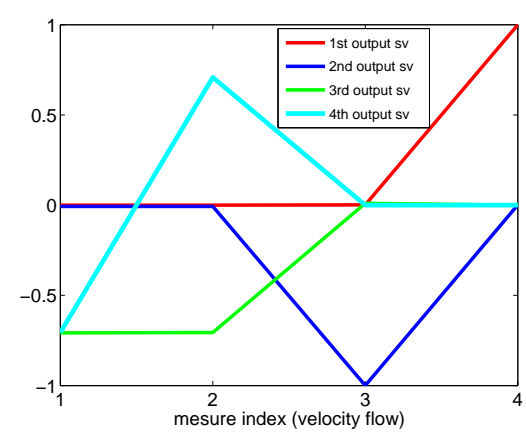

(c)

Figure 4: Results of SVD for the most likely parameters (see Table 2 and Table 3 ).

The third and fourth output singular vectors are not orthogonal to main axes:

we have represented in green the influence of the vertical hydraulic conductivity in the base zone upon the mean (or the sum) of $y_{1}$ and $y_{2}$. The only nonzero component of $v_{3}$ is its $2^{\text {nd }}$ component and $u_{3}$ has exactly two nonzero, equal components, corresponding to $y_{1}$ and $y_{2}$.

Finally we have the influence on the hydraulic conductivity in zone number 3 upon the difference between $y_{1}$ and $y_{2}$ It is represented in pale blue on the figures: $v_{4}$ has exactly 1 nonzero component and $u_{4}$ has exactly to nonzero, opposite components. Since the last singular value is almost zero, we can say that $y_{1}-y_{2}$ is almost invariable for small variations of hydraulic conductivity around this set of parameters.

The other input parameters have a negligible or null influence on the fluxes considered.

Finally we obtain almost the same conclusions as in the probabilistic study, up to the secondary influence of $k_{6}$ upon $y_{3}$. But a single local study is not enough to justify a conclusion, hence we have performed the same study for other sets of parameters.

\subsubsection{Variability of the SVD results due to the variation of the input parameters}

The other SVD results correspond to much less likely parameters. We have first computed 12 supplementary Jacobian matrices and the corresponding decompositions into singular values, by choosing the most likely value for each of the basis variables except one for which we choose either the smallest or the largest possible value. Then we have explored the vertices of the set of possible values for the hydraulic conductivities (this set is a polyhedron), that is to say we have chosen for each variable parameter either the smallest or the largest possible value, satisfying the correlations, given in Table 5 This means there are $2^{6}=64$ Jacobian matrix computations and decompositions into singular values.

$$
\begin{array}{ll}
k_{v} & \in\left\{10^{-14}, 10^{-12}\right\} \\
k_{h} & \in\left\{k_{v}, 10^{2} k_{v}\right\} \\
k_{3} & \in\left\{10^{-12}, 10^{-10}\right\} \\
k_{4} & \in\left\{10 k_{3}, 10^{3} k_{3}\right\} \\
k_{5} & \in\left\{10^{-9}, 10^{-7}\right\} \\
k_{6} & \in\left\{10 k_{5}, 10^{3} k_{5}\right\}
\end{array}
$$

Table 5: Choice of variable parameters for the deterministic analyses.

Some samples of the results obtained are given in Figures 5 through 8 . We sum up here the main tendencies we have observed. 
1. In most cases the results are similar to those of the first local study, i.e. the hierarchical list of the influences is

$$
\left(\log k_{6} \text { on } y_{4} ; \log k_{5} \text { on } y_{3} ; \log k_{v} \text { on } y_{1}+y_{2} ; \log k_{3} \text { on } y_{1}-y_{2}\right) \text {. }
$$

2. The influence of the horizontal hydraulic conductivity in the base zone $\Omega_{1} \cup \Omega_{2}$ in the sensitivity results seems to remain almost null for all possible sets of parameters.

3. The hierarchy of the influences is sometimes locally modified.

(a) Whenever $\frac{k_{5}}{k_{v}}=10^{3}$ (this is the minimal possible value), the influence of $\log k_{v}$ on $y_{1}+y_{2}$ is larger than the influence of $\log k_{5}$ on $y_{3}$. See Figures 7 and 8

(b) Whenever $\frac{k_{6}}{k_{v}}=10^{4}$ (this is the minimal possible value), the influence of $\log k_{v}$ on $y_{1}+y_{2}$ is larger than the influence of $\log k_{6}$ on $y_{4}$. See Figure 8

4. The composition of the singular vectors in the input space is sometimes modified.

(a) Whenever $\frac{k_{3}}{k_{v}}=1$ (this is the minimal possible value), we observe an influence of $\log k_{v}+\alpha \log k_{3}$ on $y_{1}+y_{2}$, where $\alpha \approx 0.5$, instead of an influence of $\log k_{v}$ on $y_{1}+y_{2}$. See Figures [6] and 8

(b) Whenever $\frac{k_{3}}{k_{v}}=10^{4}$ (this is the maximal possible value), we observe an influence of $\log k_{v}-\alpha \log k_{3}$ on $y_{1}+y_{2}$, where $\alpha \approx 0.5$, instead of an influence of $\log k_{v}$ on $y_{1}+y_{2}$. See Figure 5

(c) Whenever $k_{6}=10^{-4}$ (this is the maximal possible value), $\log k_{6}$ and $\log k_{8}$ have both an influence on $y_{4}$. See Figures [5] and [6]

(d) The parameter $k_{4}$ has a (very low) influence when $\frac{k_{4}}{k_{3}}$ and $\frac{k_{4}}{k_{v}}$ are both the smallest possible. See Figure 8

\begin{tabular}{|l|l|l|l|l|l|l|l|l|l|l|l|} 
zone $\mathrm{n}^{o}$ & 1,2 & 3 & 4 & 5 & 6 & 7 & 8 & 9 & 10 & 11 & 12 \\
\hline$k_{\alpha}$ or $\mathbf{K}_{\alpha}(\mathrm{m} / \mathrm{s})$ & $k_{h}=10^{-14}, k_{v}=10^{-14}$ & $10^{-10}$ & $10^{-9}$ & $10^{-7}$ & $10^{-4}$ & $10^{-9}$ & $210^{-7}$ & $10^{-12}$ & $10^{-11}$ & $310^{-4}$ & $310^{-5}$
\end{tabular}

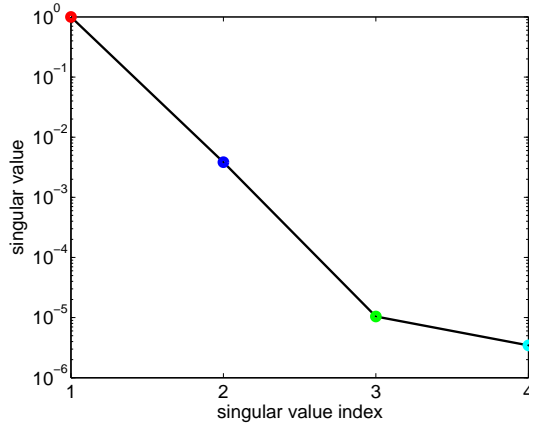

(a) $\left[3.7810^{-1} \mathrm{~m}^{3} / \mathrm{s}\right]$

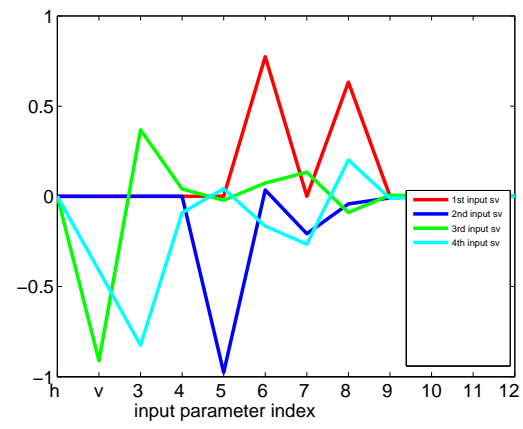

(b)

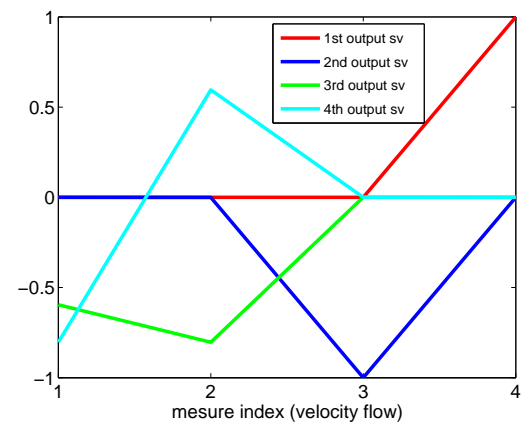

$(c)$

Figure 5: Example of SVD results, with $k_{6}$ and $\frac{k_{3}}{k_{v}}$ set at their maximal possible value. 


\begin{tabular}{|l|l|l|l|l|l|l|l|l|l|l|l|} 
zone $\mathrm{n}^{o}$ & 1,2 & 3 & 4 & 5 & 6 & 7 & 8 & 9 & 10 & 11 & 12 \\
\hline$k_{\alpha}$ or $\mathbf{K}_{\alpha}(\mathrm{m} / \mathrm{s})$ & $k_{h}=10^{-10}, k_{v}=10^{-12}$ & $10^{-12}$ & $10^{-9}$ & $10^{-7}$ & $10^{-4}$ & $10^{-9}$ & $210^{-7}$ & $10^{-12}$ & $10^{-11}$ & $310^{-4}$ & $310^{-5}$
\end{tabular}

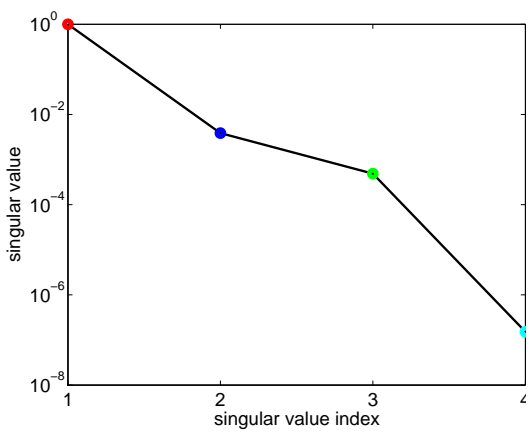

(a) $\left[3.7810^{-1} \mathrm{~m}^{3} / \mathrm{s}\right]$

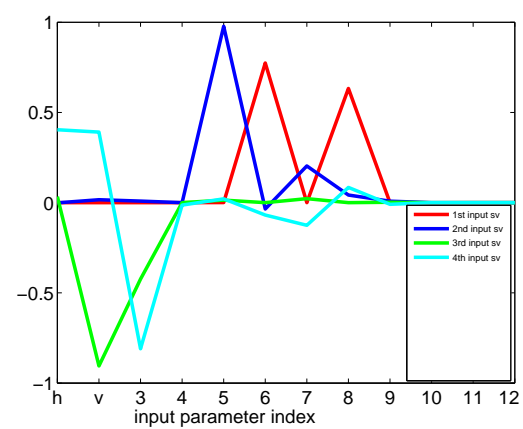

(b)

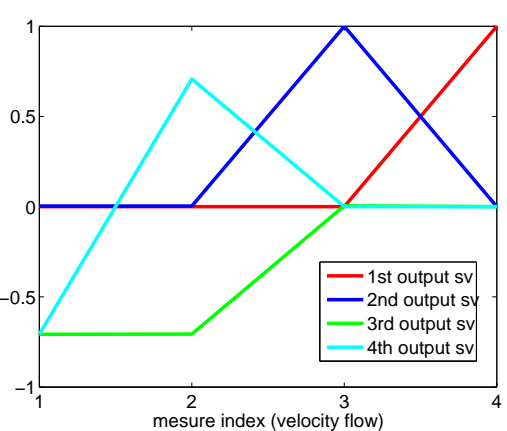

$(c)$

Figure 6: Example of SVD results, with $\frac{k_{3}}{k_{v}}$ set at its minimal possible value and $k_{6}$ set at its maximal possible value.

\begin{tabular}{|l|l|l|l|l|l|l|l|l|l|l|l|} 
zone $\mathrm{n}^{o}$ & 1,2 & 3 & 4 & 5 & 6 & 7 & 8 & 9 & 10 & 11 \\
\hline$k_{\alpha}$ or $\mathbf{K}_{\alpha}(\mathrm{m} / \mathrm{s})$ & $k_{h}=10^{-12}, k_{v}=10^{-12}$ & $10^{-10}$ & $10^{-7}$ & $10^{-9}$ & $10^{-6}$ & $10^{-9}$ & $210^{-7}$ & $10^{-12}$ & $10^{-11}$ & $310^{-4}$ & $310^{-5}$
\end{tabular}

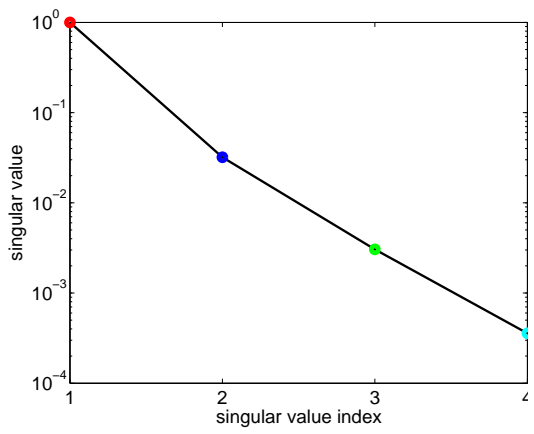

(a) $\left[9.2910^{-3} \mathrm{~m}^{3} / \mathrm{s}\right]$

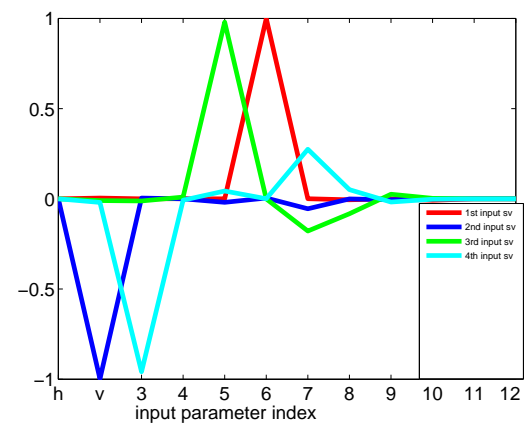

(b)

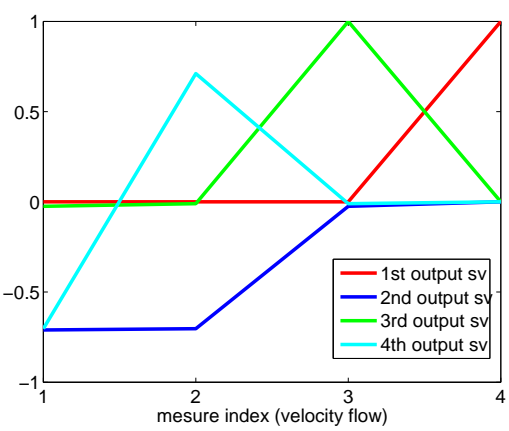

(c)

Figure 7: Example of SVD results, with $\frac{k_{5}}{k_{v}}$ set at its minimal possible value.

\subsubsection{Using the SVD results}

We consider the linearization of our problem around the most likely parameters. If the fluxes through each of the outlet channels are of the same importance, the most important input parameters to investigate well (concerning reduction of variance for example) are $\log k_{v}, \log k_{5}$ and $\log k_{6}$. The output parameter $y_{3}$ (resp. $y_{4}$ ) could be locally controlled, without modifying other output parameters, by modifying only the value of $\log k_{5}$ (resp. $\log k_{6}$ ). The output parameter $y_{1}$ could be locally controlled, without modifying other output parameters, by modifying the values of $\log k_{v}$ and $\log k_{3}$ with the same sign. The ratio between these modifications depends on the singular values following the equation $s_{3} \Delta\left(\log k_{v}\right)-s_{4} \Delta\left(\log k_{3}\right)=0$ in order to impose $\Delta y_{2}=0$. The output parameter $y_{2}$ could be locally controlled, without modifying other output parameters, by modifying the values of $\log k_{v}$ and $\log k_{3}$ with opposite sign. The ratio between these modifications depends on the singular values following the equation $s_{3} \Delta\left(\log k_{v}\right)+s_{4} \Delta\left(\log k_{3}\right)=0$ in order to impose $\Delta y_{1}=0$. Since $s_{4}$ is much smaller than $s_{3}$, it is very difficult to control independently $y_{1}$ and $y_{2}$. 


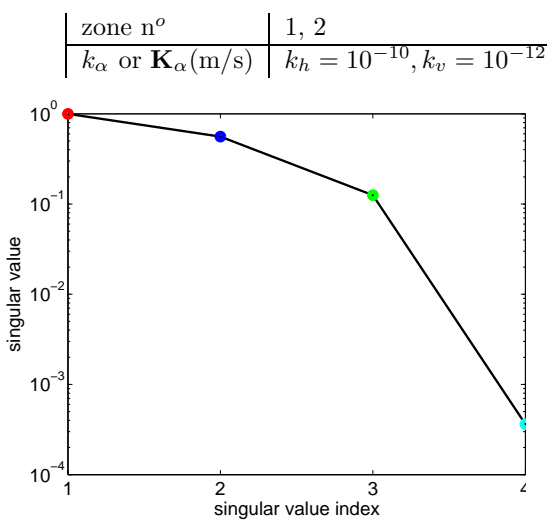

(a) $\left[1.6210^{-4} \mathrm{~m}^{3} / \mathrm{s}\right]$

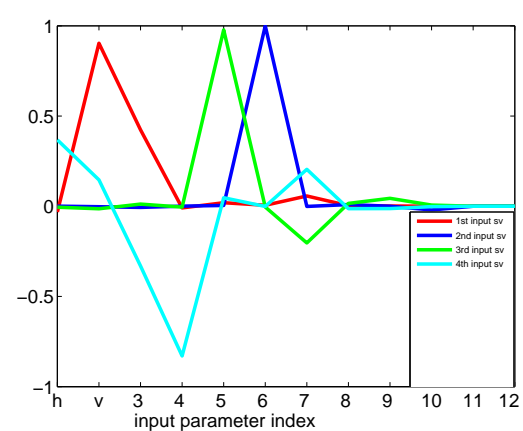

(b)

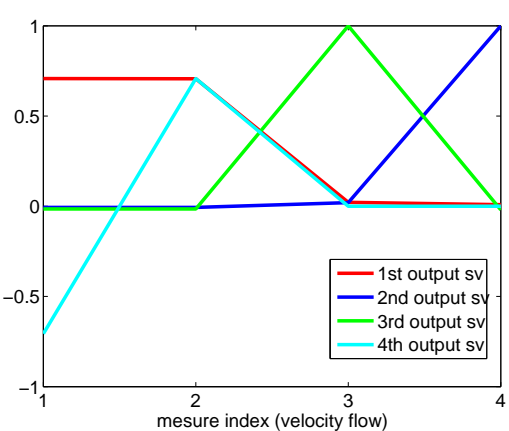

(c)

Figure 8: Example of SVD results, with $\frac{k_{3}}{k_{v}}, \frac{k_{5}}{k_{v}}, \frac{k_{6}}{k_{v}}, \frac{k_{4}}{k_{v}}$ and $\frac{k_{4}}{k_{3}}$ set at their minimal possible value.

\subsection{About computation times}

We use the three following points to predict raw comparisons of computation times for statistical and deterministic sensitivity analyses.

1. The computation cost for one row, or one column, of the Jacobian matrix $F^{\prime}(x)$ is approximately the same as the cost for applying the function $F$. Indeed, in both cases, the most computationally demanding task is to solve a linear system with the same matrix. Of course, when using direct linear solvers, it is possible to store the (possibly incomplete) factorization of the matrix and then subsequent linear system resolutions are much faster.

2. For a local analysis around $x$ using reverse mode, $F(x)$ must first be computed once, then the rows of $F^{\prime}(x)$ are computed.

3. Deterministic and statistical analyses offer roughly the same parallel possibilities.

- Independent computations: of $F$ for statistical approaches, or of row/column of $F^{\prime}$ for the deterministic approach.

- Each computation of $F$ or of row/column of $F^{\prime}$ is heavy and can be itself parallelized, for example by domain decomposition [24].

So we can compare computation times simply by comparing the number of times we have to apply function or compute one row/column of the Jacobian matrix.

For this example, $N=1000$ computations of $F$ were needed to perform the statistical analysis. Concerning the deterministic study, each local analysis leads to 1 computation of $F$ and 4 computations of rows and the complete analysis is 77 times more expensive. So the cost of the statistical analysis is about 2.5 times the cost of the complete deterministic analysis (and 200 times the cost of one single local study). 


\section{Conclusion}

In this work we have described a deterministic method for sensitivity analysis for a model that computes Darcy velocity fluxes through specified outlet channels from hydraulic conductivity parameters. We see this work as a first step towards sensitivity analysis for a model for the transport of contaminants in porous media.

The method is based on the singular value decomposition of the Jacobian matrix of the model. It yields a (weighted) hierarchical list of directions both in the space of input parameters and in the space of fluxes. It makes possible a truncated computation of the uncertainties concerning the outputs. This information being first order is only local information but it is obtained with a small calculation time. This approach to sensitivity analysis is complementary to probabilistic approaches, which are global, but more computationally demanding.

This method requires the computation of the Jacobian matrix of the model. For this the derivatives have been calculated manually (i. e. using analytic formulas) and the Jacobian matrix has been calculated row by row using the adjoint state method. The C++ code, differentiated manually, has been validated with the aid of the ADOL-C automatic differentiation library.

Several local deterministic studies have been carried out, around various sets of parameters of a wide range of probabilities. For the example studied, only a weak variability of the local influences is observed for a variation within the spectrum of the possible values of the input parameters. This is due to the weak nonlinearity of the model, assured by the choice of a logarithmic parametrization of the hydraulic conductivities. The results have been compared to those obtained from a global probabilistic study of Monte-Carlo type. The results are similar and are coherent with what might be expected for such a test case. 


\section{Acknowledgments}

This work was funded by ANDRA, the French agency for nuclear waste management, and was additionally supported by the GDR MOMAS. 


\section{References}

[1] P. Al Khoury, G. Chavent, F. Clément, and P. Hervé. Inversion of spectroscopic data, application on $\mathrm{CO}_{2}$ radiation of flame combustion. Inverse Problems in Science and Engineering, 13(3):219-240, June 2005.

[2] P. Aubert, N. Di Césaré, and O. Pironneau. Automatic differentiation in C++ using expression templates and application to a flow control problem. Comput Visual Sci, 3:197-208, 2001.

[3] C. H. Bischof, H. M. Bücker, and A. Rasch. Sensitivity analysis of turbulence models using automatic differentiation. SIAM J. on Scient. Comp., 26:510-522, 2004.

[4] F. Brezzi and M. Fortin. Mixed and Hybrid Finite Element Methods. Springer, 1991.

[5] D. G. Cacuci and M. Ionescu-Bujor. A comparative review of sensitivity and uncertainty analysis of large-scale systems - ii: Statistical methods. Nuclear Science and Engineering, 147:204-217, 2004.

[6] V. A. Cheverda, F. Clément, V. G. Khaidukov, and V. I. Kostin. Linearized inversion of multi-offset data for vertically inhomogeneous background. Journal of Inverse and Ill-Posed Problems, 5:453-484, 1998.

[7] F. Clément, N. Khvoenkova, A. Cartalade, and Ph. Montarnal. Analyse de sensibilité et estimation de paramètres de transport pour une équation de diffusion, approche par état adjoint. Technical Report 5132, INRIA, 2004.

[8] K. V. M. Fernando and H. Nicholson. Karhunen-Loève expansion with reference to singularvalue decomposition and separation of variables. Proc IEE-D, 127:204-206, 1980.

[9] J. J. Gerbrands. On the relationships between SVD, KLT and PCA. Pattern Recognition, $14: 375-381,1981$.

[10] A. Griewank. Achieving logarithmic growth of temporal and spatial complexity in reverse automatic differentiation. Optimization Methods and Software, 1:35-54, 1992.

[11] A. Griewank. Evaluating Derevatives: Principles and Techniques of Algorithmic Differentiation. Frontiers in Applied Mathematics. SIAM, Philadelphia, 2000.

[12] A. Griewank, A. Kowarz, J. Utke, O. Vogel, and A. Walther. ADOL-C: A package for the automatic differentiation of algorithms written in $\mathrm{C} / \mathrm{C}++$. documentation for ADOL-C, version 1.10.0, http://www.math.tu-dresden.de/ adol-c/adolc110.ps, July 2005.

[13] A. Griewank and A. Walther. Algorithm 799: ADOL-C: An implementation of checkpointing for the reverse and adjoint mode of computational differentiation. ACM Transactions on Mathematical Software, 26(1):19-45, March 2000.

[14] J. C. Helton and F. J. Davis. Latin hypercube sampling and the propagation of uncertainty in analyses of complex systems. Technical report, Sandia National Laboratories, November 2002 .

[15] Jon C. Helton and Freddie J. Davis. Sensitivity Analysis, chapter 6. Wiley series in probability and statistics. Wiley, 2000.

[16] R. A. Horn and C. R. Johnson. Topics in matrix analysis. Cambridge University Press, 1991.

[17] http://www.autodiff.org/.

[18] http://www.math.tu dresden.de/wir/project/revolve/. 
[19] http://www.netlib.org/lapack/.

[20] M. Ionescu-Bujor and D. G. Cacuci. A comparative review of sensitivity and uncertainty analysis of large-scale systems - i: Deterministic methods. Nuclear Science and Engineering, 147:189-203, 2004.

[21] M. Jannane, W. Beydoun, E. Crase, D. Cao, Z. Koren, E. Landa, M. Mendes, A. Pica, M. Noble, G. Roeth, S. Singh, R. Snieder, A. Tarantola, D. Trezeguet, and M. Xie. Wavelength of earth structures that can be resolved from seismic reflection data. Geophysics, 54:906-910, 1989.

[22] A. Marsh La Venue and J. F. Pickens. Application of a coupled adjoint sensitivity and kriging approach to calibrate a groundwater flow model. Water Resources Research, 28(1543-1569), June 1992.

[23] E. Laporte and P. Le Tallec. Numerical methods in sensitivity analysis and shape optimization. Birkäuser, 2003.

[24] P. Le Tallec. Domain decomposition methods in computational mechanics. Computational Mechanics Advances, 1(2), February 1994.

[25] Y. C. Liang, H. P. Lee, S. P. Lim, W. Z. Lin, K. H. Lee, and C. G. Wu. Proper orthogonal decomposition and its applications-Part I: Theory. J. Sound Vibration, 252:527-544, 2002.

[26] E. Marchand. Analyse de sensibilité déterministe pour la simulation numérique du transfert de contaminants. PhD thesis, Université Paris IX Dauphine, 2007.

[27] V. Martin. Simulation multidomaine d'un écoulement autour d'un site de stockage de déchets. PhD thesis, Université Paris IX Dauphine, 2004.

[28] J.-C. Nedelec. Mixed finite elements in $\mathbb{R}^{3}$. Numer. Math., 35:315-341, 1980.

[29] J. D. Paduano and D. R. Downing. Sensitivity analysis of digital flight control systems using singular-value concepts. J. Guidance Control Dynam., 12:297-303, 1989.

[30] P.-A. Raviart and J.-M. Thomas. A mixed finite element method for second order elliptic problems. In I. Galligani and E. Magenes, editors, Mathematical Aspects of Finite Element Methods, number 606 in Lecture Notes in Mathematics, pages 292-315. Springer, Berlin, 1977.

[31] J. E. Roberts and J.-M. Thomas. Mixed and hybrid methods. In P. G. Ciarlet and J. L. Lions, editors, Handbook of Numerical Analysis, volume II. Elsevier, 1991.

[32] S. M. Rump. Estimation of the sensitivity of linear and nonlinear algebraic problems. Lin. Algebra Appl., 153:1-34, 1991.

[33] A. Sboui. Quelques méthodes numériques robustes pour l'écoulement et le transport en milieu poreux. PhD thesis, Université Paris IX Dauphine, 2007.

[34] A. Sboui, J. Jaffré, and J. E. Roberts. A composite mixed finite element for general hexahedral grids. SISC, 2008.

[35] G. W. Stewart. On the early history of the singular value decomposition. SIAM Review, 35:551-566, 1993.

[36] M.H. Tan and J.K. Hammond. A non-parametric approach for linear system identification using principal component analysis. Mechanical Systems and Signal Processing, 21:1576-1600, 2007. 


\section{Contents}

1 Introduction $\quad 3$

2 Sensitivity analysis $\quad 4$

2.1 Probabilistic analysis . . . . . . . . . . . . . . . . . . . . . . . . . . . . . . . . . . .

2.2 Deterministic analysis . . . . . . . . . . . . . . . . . . . . . . 6

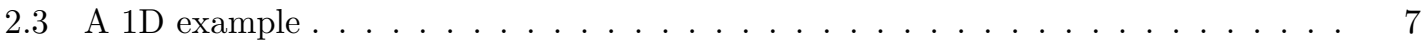

3 Differentiation $\quad \mathbf{8}$

3.1 Notation . . . . . . . . . . . . . . . . . . . . . . . . . 8

3.2 Differentiation techniques ....................... . . . . . . . . . . . . . . . . . . . . . . . . .

3.3 Verification of derivative computations . . . . . . . . . . . . . . . . . . . . . . . . . . . . . . . .

3.4 Differentiation in direct mode . . . . . . . . . . . . . . . . . . . . . . . 9

3.5 Differentiation in reverse mode . . . . . . . . . . . . . . . . . . . . . . . 10

3.5.1 The adjoint state method ...................... 10

3.5.2 Assembling the Jacobian matrix row by row . . . . . . . . . . . . . . . 11

4 Mathematical and Numerical Models 12

4.1 The Steady-state Darcy flow problem . . . . . . . . . . . . . . . . . . . . . . . 12

4.2 Discretization . . . . . . . . . . . . . . . . . . . . . . 13

4.3 Differentiation ............................ 14

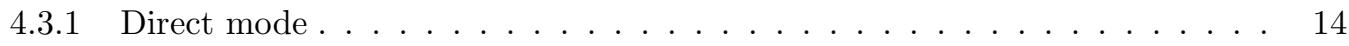

4.3 .2 Reverse mode . . . . . . . . . . . . . . . . . . . . 15

5 Numerical results $\quad 16$

5.1 Test case description . . . . . . . . . . . . . . . . . . . . . . 16

5.1.1 The computational domain . . . . . . . . . . . . . . . . 16

5.1 .2 Data for input parameters . . . . . . . . . . . . . . . 17

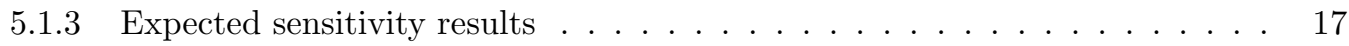

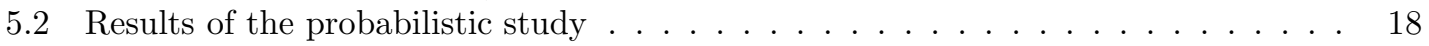

5.3 Deterministic results . . . . . . . . . . . . . . . . . . . . . 18

5.3.1 Interpretation of the SVD results . . . . . . . . . . . . . . . . 19

5.3.2 A first local study . . . . . . . . . . . . . . . . . . . . . 19

5.3.3 Variability of the SVD results due to the variation of the input parameters 20

5.3.4 Using the SVD results . . . . . . . . . . . . . . . . . . 22

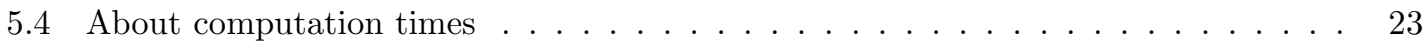

6 Conclusion $\quad 24$ 


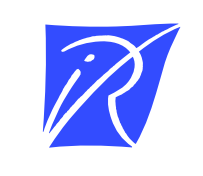

Centre de recherche INRIA Paris - Rocquencourt

Domaine de Voluceau - Rocquencourt - BP 105 - 78153 Le Chesnay Cedex (France)

Centre de recherche INRIA Bordeaux - Sud Ouest : Domaine Universitaire - 351, cours de la Libération - 33405 Talence Cedex

Centre de recherche INRIA Grenoble - Rhône-Alpes : 655, avenue de l'Europe - 38334 Montbonnot Saint-Ismier

Centre de recherche INRIA Lille - Nord Europe : Parc Scientifique de la Haute Borne - 40, avenue Halley - 59650 Villeneuve d'Ascq

Centre de recherche INRIA Nancy - Grand Est : LORIA, Technopôle de Nancy-Brabois - Campus scientifique

615, rue du Jardin Botanique - BP 101 - 54602 Villers-lès-Nancy Cedex

Centre de recherche INRIA Rennes - Bretagne Atlantique : IRISA, Campus universitaire de Beaulieu - 35042 Rennes Cedex

Centre de recherche INRIA Saclay - Île-de-France : Parc Orsay Université - ZAC des Vignes : 4, rue Jacques Monod - 91893 Orsay Cedex

Centre de recherche INRIA Sophia Antipolis - Méditerranée : 2004, route des Lucioles - BP 93 - 06902 Sophia Antipolis Cedex

INRIA - Domaine de Voluceau - Rocquencourt, BP 105 - 78153 Le Chesnay Cedex (France)

http://www.inria.fr

ISSN 0249-6399 